2013

\title{
Study of Charge-Dependent Transport and Toxicity of Peptide-Functionalized Silver Nanoparticles Using Zebrafish Embryos and Single Nanoparticle Plasmonic Spectroscopy
}

KerryJ. Lee

Old Dominion University

Lauren M. Browning

Old Dominion University

Prakash D. Nallathamby

Old Dominion University

Xiao-Hong Nancy Xu

Old Dominion University, xhxu@odu.edu

Follow this and additional works at: https://digitalcommons.odu.edu/chemistry_fac_pubs

Part of the Chemistry Commons, Pharmacology Commons, and the Toxicology Commons

\section{Repository Citation}

Lee, Kerry J.; Browning, Lauren M.; Nallathamby, Prakash D.; and Xu, Xiao-Hong Nancy, "Study of Charge-Dependent Transport and Toxicity of Peptide-Functionalized Silver Nanoparticles Using Zebrafish Embryos and Single Nanoparticle Plasmonic Spectroscopy" (2013). Chemistry \& Biochemistry Faculty Publications. 128.

https://digitalcommons.odu.edu/chemistry_fac_pubs/128

\section{Original Publication Citation}

Lee, K. J., Browning, L. M., Nallathamby, P. D., \& Xu, X. H. N. (2013). Study of charge-dependent transport and toxicity of peptidefunctionalized silver nanoparticles using zebrafish embryos and single nanoparticle plasmonic spectroscopy. Chemical Research in Toxicology, 26(6), 904-917. doi:10.1021/tx400087d 


\title{
Study of Charge-Dependent Transport and Toxicity of Peptide- Functionalized Silver Nanoparticles Using Zebrafish Embryos and Single Nanoparticle Plasmonic Spectroscopy
}

\author{
Kerry J. Lee, Lauren M. Browning, Prakash D. Nallathamby, and Xiao-Hong Nancy Xu* \\ Department of Chemistry and Biochemistry, Old Dominion University, Norfolk, Virginia 23529, United States
}

Supporting Information

\begin{abstract}
Nanomaterials possess unusually high surface area-to-volume ratios and surface-determined physicochemical properties. It is essential to understand their surface-dependent toxicity in order to rationally design biocompatible nanomaterials for a wide variety of applications. In this study, we have functionalized the surfaces of silver nanoparticles (Ag NPs, $11.7 \pm 2.7 \mathrm{~nm}$ in diameter) with three biocompatible peptides (CALNNK, CALNNS, CALNNE) to prepare positively (Ag-CALNNK NPs ${ }^{+\zeta}$ ), negatively (Ag-CALNNS NPs ${ }^{-2 \zeta}$ ), and more negatively charged NPs (Ag-CALNNE $\mathrm{NPs}^{-4 \zeta}$ ), respectively. Each peptide differs in a single amino acid at its C-terminus, which minimizes the effects of peptide sequences and serves as a model molecule to create positive, neutral, and negative charges on the surface of the NPs at $\mathrm{pH} 4-10$. We have studied their charge-dependent transport into early developing (cleavage-stage) zebrafish embryos and their effects on embryonic development using dark-field optical microscopy and spectroscopy (DFOMS). We found that all three Ag-peptide NPs passively diffused

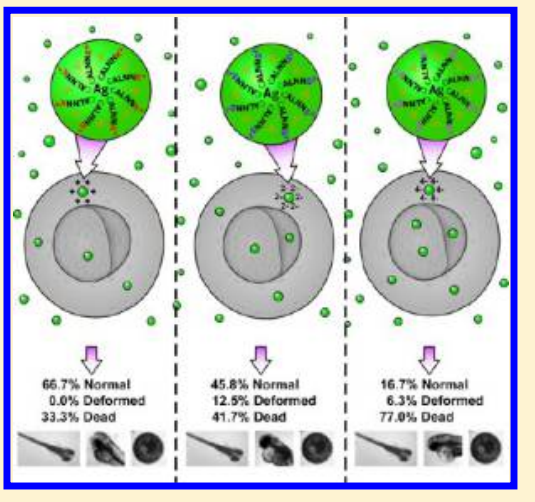
into the embryos via their chorionic pore canals, and stayed inside the embryos throughout their entire development $(120 \mathrm{~h})$, showing charge-independent diffusion modes and charge-dependent diffusion coefficients. Notably, the NPs create chargedependent toxic effects on embryonic development, showing that the Ag-CALNNK NPs ${ }^{+\zeta}$ (positively charged) are the most biocompatible while the Ag-CALNNE NPs ${ }^{-45}$ (more negatively charged) are the most toxic. By comparing with our previous studies of the same sized citrated Ag and Au NPs, the Ag-peptide NPs are much more biocompatible than the citrated Ag NPs, and nearly as biocompatible as the Au NPs, showing the dependence of nanotoxicity upon the surface charges, surface functional groups, and chemical compositions of the NPs. This study also demonstrates powerful applications of single NP plasmonic spectroscopy for quantitative analysis of single NPs in vivo and in tissues, and reveals the possibility of rational design of biocompatible NPs.
\end{abstract}

\section{INTRODUCTION}

Nanomaterials possess distinctive surface-dependent physicochemical properties and promise a wide range of applications, from consumer products to medicines. ${ }^{1-6}$ For example, the tiny sizes of nanoparticles (NPs) enable them to penetrate into living organisms, and serve as noninvasive in vivo imaging probes and drug delivery vehicles. Their high surface area-tovolume ratio allows them to conduct ultrasensitive molecular sensing for potential early disease diagnosis and to carry a larger payload of therapeutic molecules for effective drug delivery. . $^{3,7-12}$ These potential applications demand rational design of biocompatible nanomaterials, and development of effective methods to rapidly characterize their biocompatibility and toxicity.

Noble metal nanoparticles (e.g., silver nanoparticles, Ag NPs) exhibit distinctive plasmonic optical properties, which depend upon their surface sizes, shapes, dielectric constants, and surrounding environments. ${ }^{13-19}$ Individual Ag NPs possess high quantum yields (QYs) of Rayleigh scattering, which enable us to image and characterize single Ag NPs using DFOMS with a halogen lamp as an illuminator. ${ }^{7-10,12,17,18,20-24}$ Unlike fluorescence molecules or quantum dots (QDs), single Ag
NPs resist photobleaching and photoblinking, and they can serve as photostable optical probes for sensing single molecules of interest and imaging single live cells and embryos for any desired period of time with nanometer $(\mathrm{nm})$ spatial and millisecond (ms) temporal resolutions. $8,9,12,20-29$

The nanomaterials (e.g., Ag NPs) have been increasingly used in consumer products, ranging from socks, home appliances, and water treatment to disinfection. ${ }^{1,3}$ Potential release of engineered nanomaterials to ecosystems raises serious concerns about their potential environmental impacts, because their high surface area-to-volume ratios could lead to high reactivity, and potentially cause adverse effects on living organisms. These concerns have inspired a wide variety of studies on nanotoxicity. ${ }^{8,25-28,30-33}$

Currently, toxicities of nanomaterials are primarily studied using conventional cellular toxicology assays, and their doses and physicochemical properties (sizes, shapes and surface properties) are not characterized in vivo in situ in real time. ${ }^{34-37}$ Furthermore, unpurified, unstable, or functionalized nanoma-

Received: March 4, 2013

Published: April 26, 2013 
terials are widely used in the studies without in situ characterization of their physicochemical properties. ${ }^{38-40}$ Notably, nanomaterials contain various residual chemicals, which depend upon their preparation protocols. These residual chemicals could cause various degrees of toxicity. Nanomaterials also need special care in order to remain stable (nonaggregated) in the medium of the study. Unfortunately, many studies did not consider the effects of their stability and residual chemicals involved in preparation of nanomaterials, leading to inconclusive and contradictory results. ${ }^{34-37}$

To study surface-dependent nanotoxicity, one must prepare stable and purified nanomaterials and develop in vivo assays and imaging tools to quantitatively determine doses, sizes, and surface properties of nanomaterials in situ in real time. Otherwise, unstable NPs could aggregate to become larger NPs and precipitate out of the solution, leading to lower doses. Unpurified NPs could provide various unexpected chemicals that would cause wide ranges of toxicities. Thus, one would unknowingly study size-, dose-, and chemical-contaminateddependent toxicity, instead of surface-dependent toxicity.

Zebrafish embryos are superior in vivo model organisms over other model organisms (e.g., mouse, rat, human). ${ }^{41-43}$ Notably, the embryos develop ex utero and they are optically transparent, which enables real-time visualization of developmental processes and related pathological and mal-development phenotypes, and direct study of transport and effects of NPs on embryonic development in vivo in real time. Zebrafish embryos complete their early development rapidly $(120 \mathrm{~h})$, and each developmental stage is well-defined. Massive amount of embryos can be produced overnight at very low cost. Therefore, zebrafish embryos can potentially serve as high-throughput ultrasensitive in vivo assays and as effective aquatic model organisms for screening of toxicity of a wide variety of nanomaterials, and monitoring of their potential release into ecosystems (e.g., rivers, ocean).$^{24-29}$

In our previous studies, ${ }^{24-29}$ we used early developing zebrafish embryos (cleavage-stage) to study the size- and chemical-composition-dependent transport, biocompatibility, and toxicity of various-sized $\mathrm{Ag}$ NPs $(11.6 \pm 3.5,41.6 \pm 9.1$, and $95.4 \pm 16.0 \mathrm{~nm})$ and Au NPs $(11.6 \pm 0.9 \mathrm{~nm} ; 86.2 \pm 10.8$ $\mathrm{nm})$. In this study, we synthesize and characterize various charged Ag-peptide NPs $(11.7 \pm 2.7 \mathrm{~nm})$, and study their charge-dependent transport into the embryos and their effects on embryonic development over $120 \mathrm{~h}$, aiming to better understand surface-dependent transport and nanotoxicity, and to rationally design biocompatible NP imaging probes.

\section{MATERIALS AND METHODS}

Synthesis and Characterization of Ag-Peptide NPs. We first synthesized the citrated $\mathrm{Ag}$ NPs by mixing $\mathrm{AgClO}_{4}(10 \mathrm{mM}$ in nanopure, deionized (DI) water, $2.5 \mathrm{~mL}$ ) with a freshly prepared icecold solution $(247.5 \mathrm{~mL})$ of sodium citrate $(3 \mathrm{mM})$ and sodium borohydride $(10 \mathrm{mM})$ with stirring overnight, as we described previously. ${ }^{27,44}$ Polyethylene glycol (PEG-20) was added to the colloidal Ag NPs to have the final PEG concentration of $0.05 \%$ (weight/volume, w/v), and stirred for $45 \mathrm{~min}$ to stabilize the NPs. We then filtered the solution using $0.22 \mu \mathrm{m}$ filters. The solution $(20 \mathrm{~mL}$ of colloidal Ag NPs containing PEG, $0.05 \% \mathrm{w} / \mathrm{v}$ ) was then mixed dropwise with each type of peptide $(0.38 \mathrm{mM}$ in $0.5 \mathrm{mM}$ PBS buffer with $1.5 \mathrm{mM} \mathrm{NaCl}, \mathrm{pH} 7.0$ ), CALNNK, CALNNS, or CALNNE, respectively. ${ }^{45,46}$ The mixtures were continuously shaken at $4{ }^{\circ} \mathrm{C}$ for $12 \mathrm{~h}$ to achieve the $\mathrm{S}_{\mathrm{N}} 2$ replacement reaction of the $-\mathrm{SH}$ group of the peptides with citrates adsorbed on the surface of the NPs to prepare

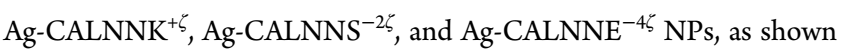
in Figure 1.

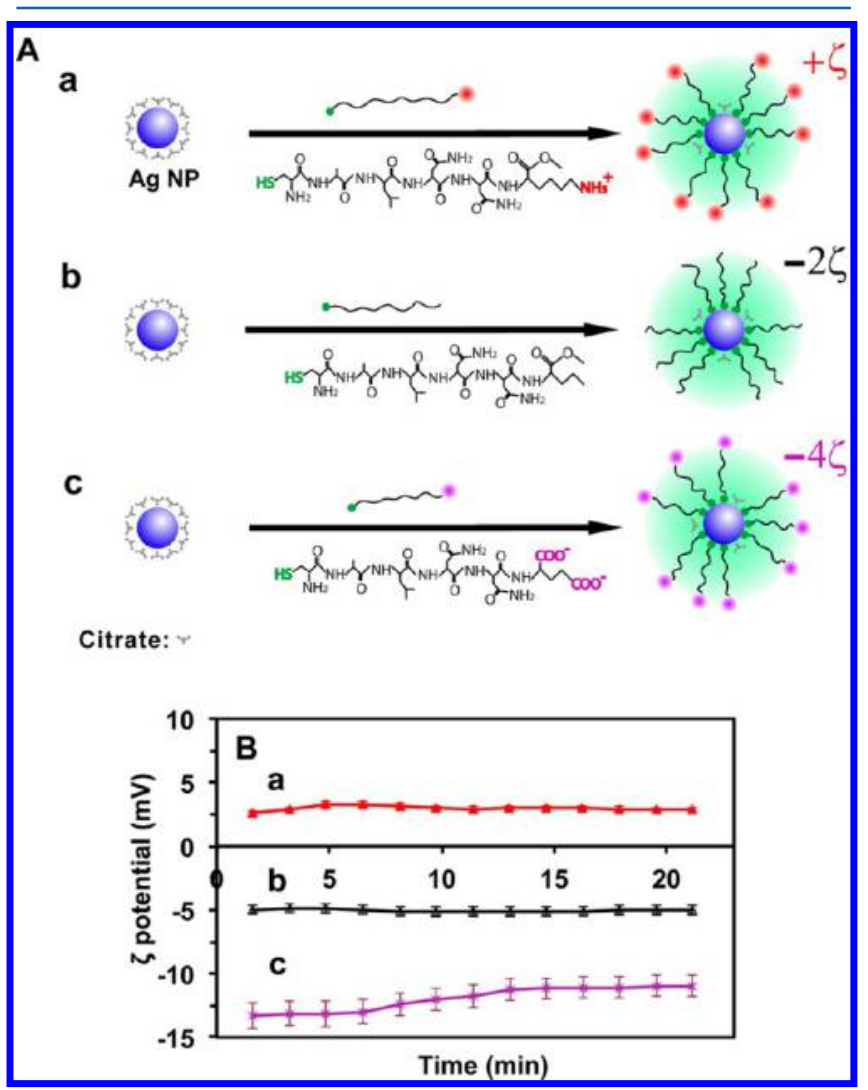

Figure 1. Synthesis and characterization of Ag-peptide NPs. (A) The Ag-peptide NPs: (a) Ag-CALNNK NPs ${ }^{+\zeta}$, (b) Ag-CALNNS NPs ${ }^{-2 \zeta}$, and (c) Ag-CALNNE NPs ${ }^{-4 \zeta}$ are prepared by functionalizing Ag NPs with a given type of peptide (either CALNNK, CALNNS, or CALNNE) using the interaction of the thiol group $(-\mathrm{SH})$ of cysteine with the surface of Ag NPs via the replacement reaction of the peptide with citrate. (B) Plots of zeta potentials of the NPs in egg water versus time show that their zeta potentials remain unchanged over time at (a) $3.0 \pm 0.2$, (b) $-5.0 \pm 0.1$, and (c) $-11.9 \pm 0.9 \mathrm{mV}$.

The Ag-peptide NPs were washed three times with nanopure DI water using centrifugation to remove the residual chemicals involved in synthesis. The NPs in the pellets were resuspended in egg water (1.0 $\mathrm{mM} \mathrm{NaCl}$ in DI water, embryonic medium), which were used to characterize their stability and to study their transport into/in embryos and their effects on embryonic development. The supernatant collected from the last washing step was used to incubate with embryos over $120 \mathrm{~h}$, which served as a control experiment to determine the effects of any possible trace chemicals involved in NP synthesis on embryonic development.

We resuspended the pellet of each type of purified Ag-peptide NPs in egg water and characterized their sizes, zeta-potentials, concentrations, and optical properties using high-resolution transmission electron microscope (HRTEM, JEOL, JEM-2100 F), dynamic light scattering (DLS, Nicomp 380ZLS particle sizing and system), UV-vis absorption spectroscopy (Hitachi U2010), and DFOMS (Figures 2-4 and Figures S1 and S2 in the Supporting Information), respectively. We characterized various concentrations of Ag-peptide NPs in egg water over $120 \mathrm{~h}$ to determine their stability and "solubility" (nonaggregation) in egg water over the entire period of embryonic development $(120 \mathrm{~h})$. The purified and stable Ag-peptide NPs were used to study their diffusion into embryos and their effects on embryonic development.

We have fully described the designs and applications of our DFOMS for real-time imaging and spectroscopic characterization of 


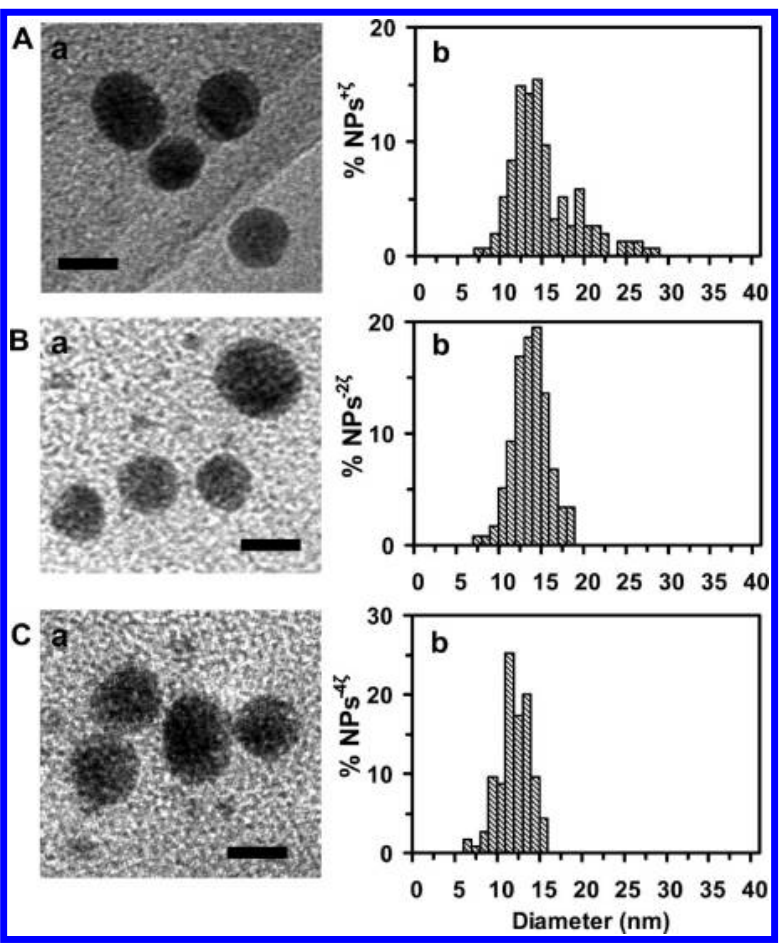

Figure 2. Characterization of sizes and shapes of single Ag-peptide NPs using HR-TEM: (a) HR-TEM images and (b) histogram of size distributions of (A) Ag-CALNNK NPs ${ }^{+\zeta}$, (B) Ag-CALNNS NPs ${ }^{-2 \zeta}$, and (C) Ag-CALNNE NPs ${ }^{-4 \zeta}$ show their average diameters of $11.5 \pm$ $2.9,12.3 \pm 2.7$, and $11.3 \pm 2.5 \mathrm{~nm}$, respectively. The scale bars are 10 $\mathrm{nm}$.

single NPs in solutions, single live cells, and embryos, and for single molecule detection. ${ }^{7-10,12,17,20-29,47}$ In this study, a dark-field microscope coupled with a CCD camera (EMCCD, Coolsnap HQ2 or Micromax) and a spectrograph (SpectraPro-150, Roper Scientific) or Multispectral Imaging System (MSIS, N-MSI-VIS-FLEX, CRi, Hopkiton, MA) were used to image and characterize LSPR spectra of single Ag-peptide NPs. The MSIS is an integrated system of a CCD camera (SonyICX 285) and liquid crystal tunable filter. ${ }^{10,12,26,28,48,49}$ DFOMS-MSIS can simultaneously acquire dark-field plasmonic optical images and spectra of a massive amount of individual NPs with spectral resolution of $1 \mathrm{~nm}$, and enable high-throughput spectral analysis and characterization of single NPs. The dark-field microscope is equipped with a dark-field condenser (oil, 1.43-1.20), a microscope illuminator (Halogen lamp, $100 \mathrm{~W}$ ), and a $100 \times$ objective (Plan fluor $100 \times$, N.A. $0.5-1.3$ ).

The customized peptides (98\% purity, ChemPep, Inc.) and all other chemicals (Sigma) were purchased and used as received. The DI water (18 M 2 , Barnstead) was used to prepare solutions and rinse glassware.

Breeding and Monitoring of Development of Zebrafish Embryos. We housed wild-type adult zebrafish (Aquatic Ecosystems) in a stand-alone system (Aquatic Habitats) and maintained and bred them as described previously. ${ }^{25-29,50}$ Briefly, we placed $2-3$ pairs of mature zebrafish into a clean 10-gallon breeding tank and used a light $(14 \mathrm{~h})$-dark $(10 \mathrm{~h})$ cycle to trigger the breeding and fertilization of embryos. The cleavage-stage embryos $(0.75-2.25 \mathrm{hpf})$ were collected, transferred to Petri dishes, and thoroughly rinsed with egg water to remove the surrounding debris. The washed embryos were then used for real-time imaging of the diffusion and transport of single Agpeptide NPs into/in embryos and for quantitative study of their effects on embryonic development. All experimental procedures involving embryos and zebrafish were performed in compliance with the IACUC guidelines.

In Vivo Real-Time Imaging of Diffusion and Transport of Single Ag-Peptide NPs into/in Embryos. The cleavage-stage embryos were incubated with a given type of Ag-peptide NPs (0-0.6

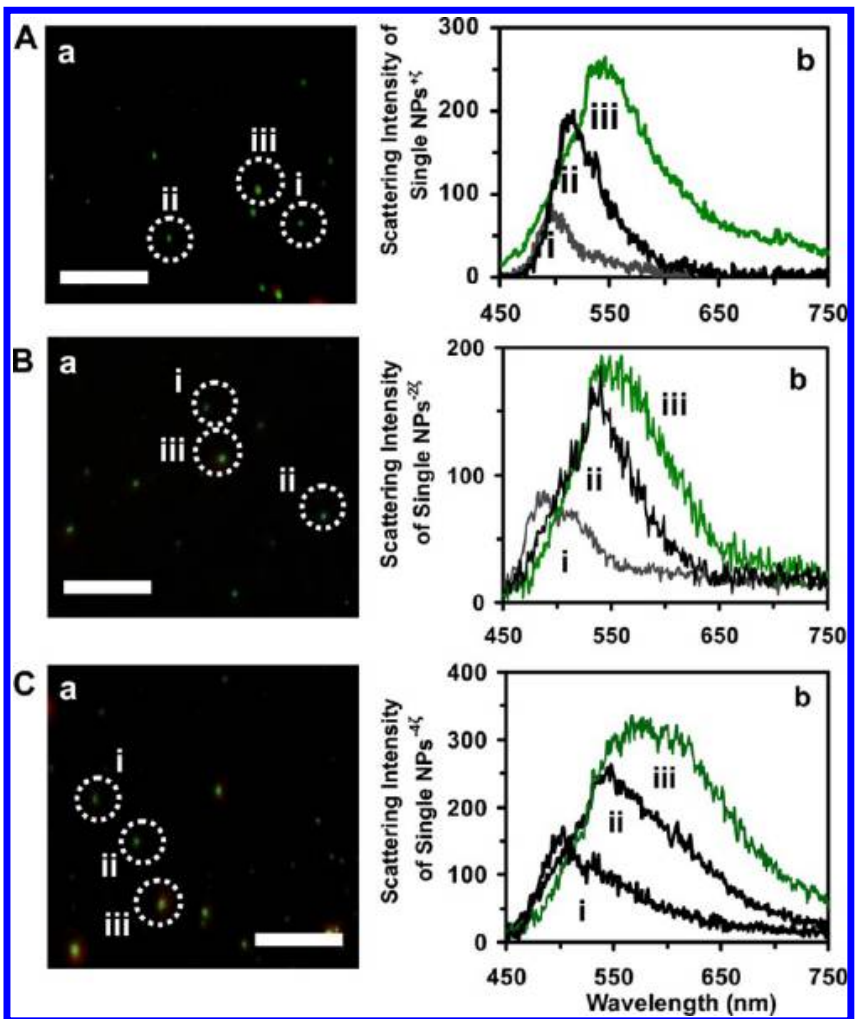

Figure 3. Study of plasmonic optical properties of single Ag-peptide NPs using DFOMS. (a) Dark-field optical images and (b) LSPR spectra of single (A) Ag-CALNNK NPs ${ }^{+\zeta}$, (B) Ag-CALNNS NPs ${ }^{-2 \zeta}$, and (C) Ag-CALNNE NPs ${ }^{-4 \zeta}$ show plasmonic green NPs with the following $\lambda_{\max }$ (full-width-at-half-maximum, fwhm): (A) (i) 508 (72); (ii) 544 (93); (iii) 585 (99); (B) (i) 508 (68); (ii) 560 (88); (iii) 581 (101); (C): (i) 506 (69); (ii) 555 (98); (iii) 595 (111) nm; respectively. The scale bars are $2 \mu \mathrm{m}$, which show the distances among NPs, but not their sizes due to optical diffraction limit.

$\mathrm{nM}$ ), either Ag-CALNNK ${ }^{+\zeta},{\mathrm{Ag}-\mathrm{CALNNS}^{-2 \zeta} \text {, or Ag-CALNNE }}^{-4 \zeta}$ NPs, in a microchamber. The diffusion and transport of single NPs into/in embryos were imaged in real time using our DFOMS (Figure 5 and Figures S3-S5 in the Supporting Information), aiming to study the charge-dependent transport and diffusion modes of single NPs. As we described previously, ${ }^{25-28}$ we acquired localized surface plasmon resonance (LSPR) spectra of single Ag-peptide NPs using DFOMSMSIS (Figures 3, 9-11), and used their distinctive plasmonic green colors to identify and distinguish them from embryonic debris and zebrafish tissues, which appear white under dark-field illumination.

Study of Dose- and Charge-Dependent Toxicity of AgPeptide NPs. We incubated the cleavage-stage embryos with a dilution series of a given type of the Ag-peptide NPs, either Ag$\mathrm{CALNNK}^{+\zeta}, \mathrm{Ag}^{-\mathrm{CALNNS}^{-2 \zeta}}$, or Ag-CALNNE ${ }^{-4 \zeta} \mathrm{NPs}(2.0 \mathrm{~mL}$ per well of $0,0.1,0.2,0.4$, and $0.6 \mathrm{nM}$ ) in 24-well plates (4 embryos/well) for $120 \mathrm{~h}$. The molar concentrations of Ag-peptide NPs (number of single NPs per volume) were calculated as we described previously. ${ }^{25-28,51}$ We conducted the control experiments by incubating the embryos (4 embryos/well) with egg water alone, the supernatants collected from the last washing of the Ag-peptide NPs, and the peptide alone (in the absence of NPs) in three rows of the same well plate as those embryos incubated with the NPs. Three replicates (two additional sets of four embryos in each given concentration in each well) were performed simultaneously. Thus, the 12 embryos in three wells for each concentration and control were studied for each run of the experiment. Each experiment was carried out four times, and a total number of 48 embryos (12 replicates of 4 embryos for each concentration) were studied for each NP concentration and each control experiment to gain representative statistics (Figures 7 and 8). 


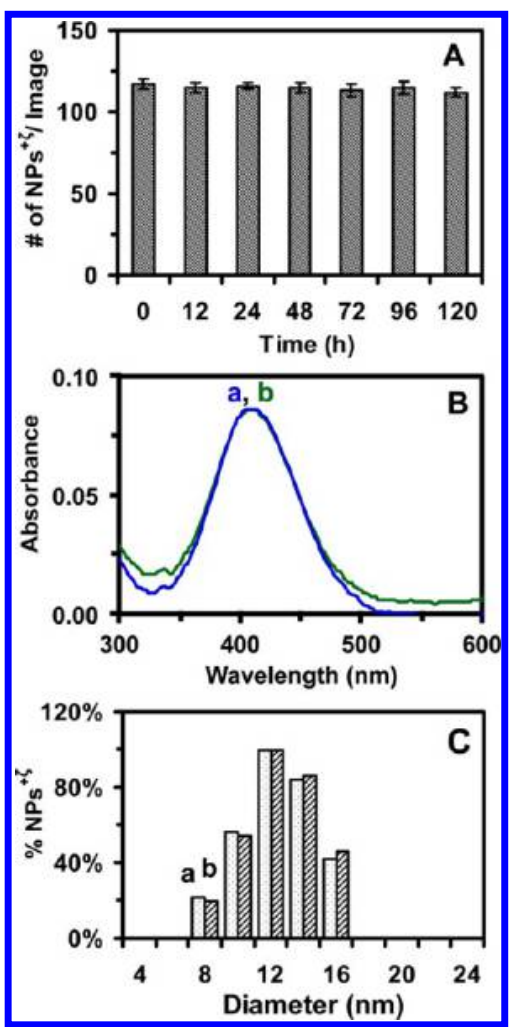

Figure 4. Characterization of stability of Ag-CALNNK $\mathrm{NPs}^{+\zeta}$ in egg water $\left(C_{\mathrm{NPs}}=0.6 \mathrm{nM}\right)$ for $120 \mathrm{~h}$. (A) The average number of NPs per image at $0,12,24,48,72,96$, and $120 \mathrm{~h}$ is $117 \pm 3,115 \pm 3,116 \pm 2$, $115 \pm 3,113 \pm 4,115 \pm 4$, and $112 \pm 3$, respectively. The 20 images are acquired at each given time using DFOMS. (B) UV-vis absorption spectra of the NPs dispersed in egg water at $28.5^{\circ} \mathrm{C}$ for (a) 0 and (b) $120 \mathrm{~h}$ show that its peak absorbance of 0.083 at $406 \mathrm{~nm}$ (fwhm $=100$ $\mathrm{nm}$ ) remains unchanged for $120 \mathrm{~h}$. (C) Histograms of size distributions of the NPs dispersed in egg water and measured by DLS show their average diameters of (a) $11.2 \pm 2.5$ and (b) $11.3 \pm 2.6$ $\mathrm{nm}$, for 0 and $120 \mathrm{~h}$, respectively. The study of stability of AgCALNNS NPs ${ }^{-2 \zeta}$ and Ag-CALNNE NPs ${ }^{-4 \zeta}$ in egg water for $120 \mathrm{~h}$ is shown in Figures $\mathrm{S} 1$ and $\mathrm{S} 2$ in the Supporting Information, respectively.

The microwell plates with the embryos were incubated in a water bath at $28.5^{\circ} \mathrm{C}$ in the dark for $120 \mathrm{~h}$. The developing embryos in the plates were imaged at 2, 24, 48, 72, 96, and $120 \mathrm{hpf}$ using an inverted microscope (Zeiss Axiovert) equipped with a CCD camera (CoolSnap, Roper Scientific).

Quantitative Imaging and Analysis of Single Ag-Peptide NPs Embedded in Zebrafish. The cleavage-stage embryos were incubated with the given concentrations $(0-0.6 \mathrm{nM})$ of each type of

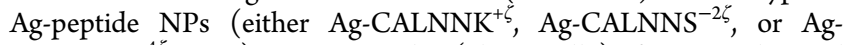
CALNNE $^{-4 \zeta}$ NPs) continuously (chronically) for $120 \mathrm{~h}$, and developed to normal or deformed zebrafish. The normal and deformed zebrafish were carefully rinsed with DI water to remove any external NPs, fixed using a tissue processor (Microm STP-120 Spin, Thermo Fisher Scientific), embedded with paraffin using a tissue embedding center (Shandon Histocenter 3 Embedding Center), and sectioned to prepare thin-layer tissue samples $(1-2 \mu \mathrm{m}$ thickness) using a microtome (HM360 rotary microtome, Thermo Fisher Scientific), as we described previously. ${ }^{2-28}$ The number of individual Ag-peptide NPs embedded in the tissues of interest were quantitatively determined using their LSPR spectra acquired by DFOMS-MSIS (Figures 9-11). A minimum of three slices of each given tissue were analyzed for each measurement.

Statistical Analysis. For characterization of sizes, LSPR spectra, and stability of single Ag-peptide NPs using HRTEM and DFOMS, we studied 300 individual NPs for each type of Ag-peptide NPs with a minimum of 100 NPs per measurement. For real-time imaging of transport and diffusion of single Ag-peptide NPs into and in embryos, we characterized a minimum of 12 embryos for each given concentration. For the study of dose- and charge-dependent effects of the Ag-peptide NPs on embryonic development, we studied a total number of 48 embryos with 12 replicates of 4 embryos per measurement for each NP concentration and each control experiment.

We used a conventional statistical analysis method (ANOVA) to analyze and determine the significant differences of the embryos that developed to normal or deformed zebrafish, or became dead, as they were incubated with various concentrations of each given type of $\mathrm{Ag}$ peptide NPs $(0-0.6 \mathrm{nM})$. We found significant dose- and chargedependent nanotoxicity for each given type of Ag-peptide NPs with 95\% confidence level $(P=0.05)$. In this study, we characterized the effects of Ag-peptide NPs on embryonic development at single embryo and single NP resolution, and observed the rare events of interest (deformed zebrafish in Figure 6D-F), which otherwise would be buried under ensemble measurements. The large number of single Agpeptide NPs and single embryos were studied to offer representative statistics to depict the ensemble properties of NPs and their effects on embryonic development at single NP and single embryo resolutions.

\section{RESULTS AND DISCUSSION}

Synthesis and Characterization of Stable and Purified Ag-Peptide NPs. We synthesized, purified, and characterized three different charged Ag-peptide NPs by functionalizing the surface of Ag NPs with three biocompatible peptides (CALNNK, CALNNS, or CALNNE), respectively, as described in Figure 1A and in Materials and Methods. ${ }^{45,46}$ We purposely designed the nearly identical peptides as surface functional groups for each type of NPs, aiming to minimize the potential effects of various surface functional groups on embryonic development. Each peptide consists of 5 identical amino acids with the identical sequence (CALNN). Each peptide differs in a single amino acid (lysine, serine, and glutamine with $\mathrm{p} K_{\mathrm{a}}$ of $10.8,16$, and 4.0 , respectively) at its C-terminus, which offers positive, neutral, and negative charges at $\mathrm{pH} 4-10$. Each peptide has a cysteine on its $\mathrm{N}$-terminus, and each cysteine has a thiol group $(-\mathrm{SH})$. The peptide was attached onto the $\mathrm{Ag}$ NPs by the interaction of the $-\mathrm{SH}$ of cysteine with the surface of Ag NPs via the $S_{N} 2$ replacement reaction of $-\mathrm{SH}$ with the citrate adsorbed on the $\mathrm{Ag} \mathrm{NPs}$, which generated $\mathrm{Ag}$ CALNNK, Ag-CALNNS, and Ag-CALNNE NPs, respectively. The NPs were thoroughly washed with DI water using centrifugation to prepare the purified NPs.

We characterized the surface charges of the purified Agpeptide NPs in egg water by measuring their surface zeta potentials $(\zeta)$ over time, which show $(3.0 \pm 0.2),-(5.0 \pm 0.1)$, and $-(11.9 \pm 0.9) \mathrm{mV}$ for the Ag-CALNNK, Ag-CALNNS, and Ag-CALNNE NPs (Figure 1B), respectively. The results indicate that Ag-CALNNK NPs ${ }^{+\zeta}$ have four times fewer charges than Ag-CALNNE NPs ${ }^{-4 \zeta}$, while the Ag-CALNNS NPs ${ }^{-2 \zeta}$ exhibit negative charges (twice amount of the charges as AgCALNNK NPs ${ }^{+\zeta}$ ), instead of the neutral. Note that the same amount of each peptide was attached onto the surface of the $\mathrm{Ag}$ NPs, as we characterized by measuring the amount of each peptide remaining in the solution (supernatant) after the functionalization. Therefore, the results suggest that the replacement of the given peptides with citrate molecules on the Ag NPs might be incomplete and some negatively charged citrates might remain on the NPs. In this study, we purposely prepared the Ag-peptide NPs with the lower surface charges, aiming to compare their toxic effects with the same sized citrated $\mathrm{Ag}$ and $\mathrm{Au}$ NPs with similar zeta potential of $-5 \mathrm{mV}$ in egg water, and to determine the sensitivity of cleavage-stage 


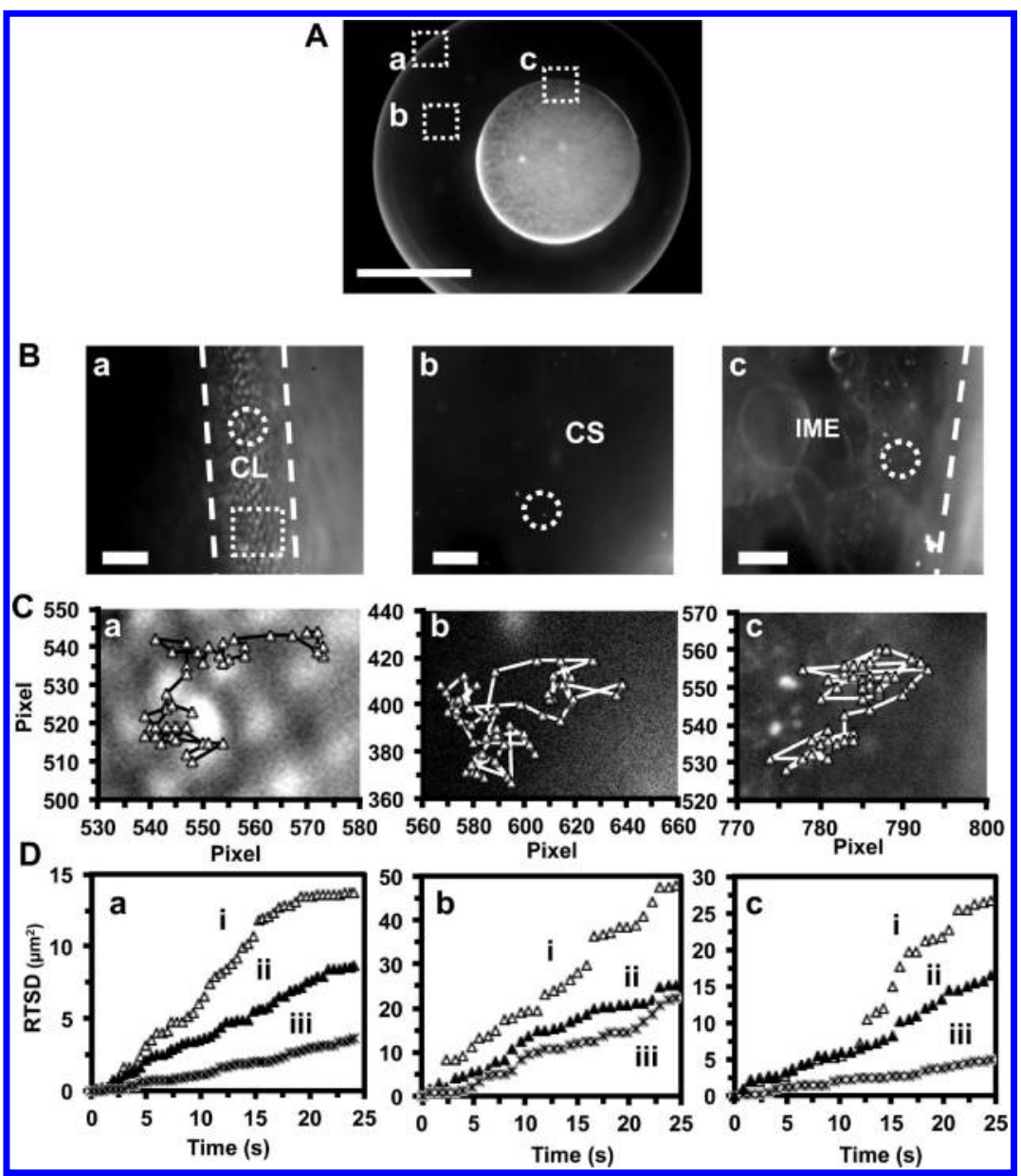

Figure 5. Real-time study of charge-dependent transport and diffusion modes of single Ag-peptide NPs $\left(C_{\mathrm{NPs}}=0.6 \mathrm{nM}\right)$ in chorion layers $(\mathrm{CL})$, chorion space (CS), and inner mass of the embryo (IME). (A) The cleavage-stage embryo shows (a) CL (the interface of chorion space and egg water), (b) CS, and (c) the interface of CS and IME, as highlighted by squares. (B) Optical images show the diffusion of single green Ag-CALNNK $\mathrm{NPs}^{+\zeta}$ (as circled) and (C) their real-time diffusion trajectories in (a) CL (as highlighted by two dashed lines); the square highlights the arrays of (white dots) chorionic pore canals (CPCs). The pores have diameters of $0.5-0.7 \mu \mathrm{m}$ and distances among neighboring pores at $1.5-2.5 \mu \mathrm{m}$; (b) CS; (c) IME. The real-time optical images and diffusion trajectories of single green Ag-CALNNS NPs ${ }^{-2 \zeta}$ and Ag-CALNNE NPs ${ }^{-4 \zeta}$ are shown in Figures S3-S5 in the Supporting Information. (D) Plots of RTSD versus diffusion time of single green: (i) Ag-CALNNK NPs ${ }^{+\zeta}$, (ii) Ag-CALNNS $\mathrm{NPs}^{-2 \zeta}$, and (iii) Ag-CALNNE NPs ${ }^{-4 \zeta}$ show (a) constrained random Brownian motion in CL with diffusion coefficients of $(\mathrm{i})(1.7 \pm 0.4) \times 10^{-9}$, (ii) $(9.3 \pm 2.5) \times 10^{-10}$, and (iii) $(3.4 \pm 1.0) \times 10^{-10} \mathrm{~cm}^{2} \mathrm{~s}^{-1}$; (b) simple random Brownian motion in CS with diffusion coefficients of (i) (4.6 \pm $0.9) \times 10^{-9}$, (ii) $(2.8 \pm 0.3) \times 10^{-9}$, and (iii) $(2.1 \pm 1.5) \times 10^{-9} \mathrm{~cm}^{2} \mathrm{~s}^{-1}$; and (c) simple random Brownian motion at the interface of CS and IME with diffusion coefficients of (i) $(3.0 \pm 1.5) \times 10^{-9}$, (ii) $(1.5 \pm 0.4) \times 10^{-9}$, and (iii) $(4.6 \pm 0.7) \times 10^{-10} \mathrm{~cm}^{2} \mathrm{~s}^{-1}$. Scale bars are (A) 400 and (B) 10 $\mu \mathrm{m}$, and each pixel in (C) is $0.067 \mu \mathrm{m}$. The temporal resolution of sequential optical images for diffusion trajectory measurements in (b) is $100 \mathrm{~ms}$.

embryos as in vivo assays for study of nanotoxicity. Furthermore, we cannot study the neutral NPs, because surface charges of the NPs are essential for them to avoid aggregations and to suspend in the solution.

We characterized the sizes and shapes of the Ag-peptide NPs using HR-TEM (Figure 2), showing spherical NPs with $11.5 \pm$ $2.9,12.3 \pm 2.7$, and $11.3 \pm 2.5 \mathrm{~nm}$ diameters for Ag-CALNNK $\mathrm{NPs}^{+\zeta}$, Ag-CALNNS $\mathrm{NPs}^{-2 \zeta}$, and Ag-CALNNE $\mathrm{NPs}^{-4 \zeta}$, respectively. We studied plasmonic optical properties and LSPR spectra of single Ag-peptide NPs in egg water using DFOMS (Figure 3), which shows plasmonic green NPs with peak wavelengths $\left(\lambda_{\max }\right)$ ranging from 506 to $595 \mathrm{~nm}$. The distribution of LSPR spectra of single Ag-peptide NPs is attributed to the various sizes and surface morphologies of single Ag NPs. Their sizes can be determined in situ in real time using their LSPR spectra via calibration approaches (optical nano ruler), as we reported previously. ${ }^{17,21,24,27}$ Notably, functionalization of the Ag NPs with the peptides led to the red shift of their LSPR spectra from blue to the green, due to the decrease of their surface reflectivity and dielectric constant, and increase of their surface sizes, as those we reported previously. ${ }^{7,9,10,12}$ It also led to about one order of magnitude weaker scattering intensity of single NPs, making it even more challenging to image them.

We characterized the stability and "solubility" of NPs (maximum concentrations of non-aggregated NPs) in the egg water for $120 \mathrm{~h}$ using DFOMS, UV-vis absorption spectroscopy, and DLS. The results in Figure 4 and Figures S1 and S2 in the Supporting Information show that the number of each type of Ag-peptide NPs suspended in egg water $(0.6 \mathrm{nM})$, their UV-vis absorption spectra, and their sizes remain unchanged for $120 \mathrm{~h}$, indicating that the NPs are very stable (nonaggregated) in egg water over $120 \mathrm{~h}$ (entire duration of embryonic development). Notably, the sizes of Ag-CALNNK $\mathrm{NPs}^{+\zeta},{\mathrm{Ag}-\mathrm{CALNNS} \mathrm{NPs}^{-2 \zeta} \text {, and Ag-CALNNE NPs }}^{-4 \zeta}$ in egg water measured by DLS (Figure 4 and Figures S1 and S2 in the 


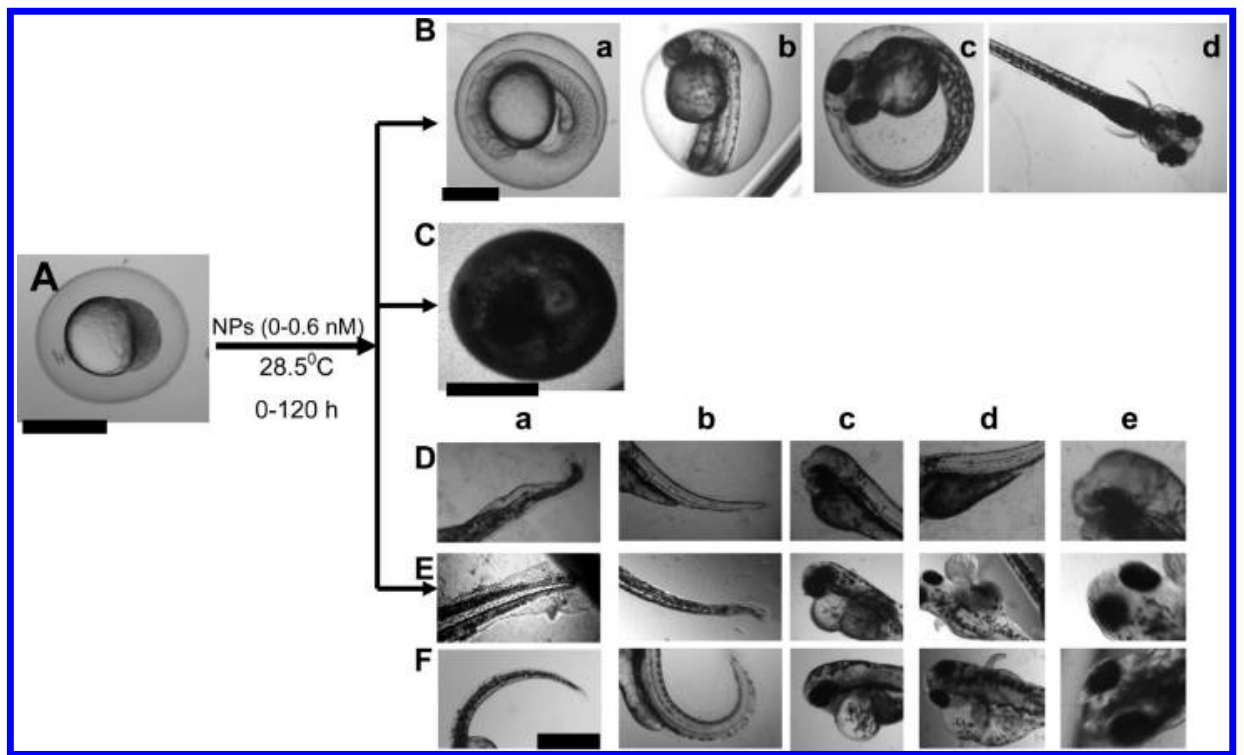

Figure 6. Optical images of: (A) Cleavage-stage embryos $(0.75-2.25 \mathrm{hpf})$ that are used to incubate with the NPs $(0-0.6 \mathrm{nM})$ over $120 \mathrm{~h}$. (B) Normally developing zebrafish embryos: (a) late segmentation stage (24 hpf); (b) hatching stage (48 hpf); (c) protruding mouth pharyngula stage (72 hpf); and (c) fully developed larvae (120 hpf). (C) Dead embryos. (D-F) Deformed zebrafish for (D) Ag-CALNNK NPs ${ }^{+\zeta}$, (E) Ag-CALNNS $\mathrm{NPs}^{-2 \zeta}$, and (F) Ag-CALNNE NPs ${ }^{-4 \zeta}$ incubated with the embryos over $120 \mathrm{~h}$ show (a) finfold abnormality, (b) tail/spinal cord flexure, (c) cardiac malformation, (d) yolk sac edema, (e) no eye in (D) and small eyes in (E) and (F). Scale bars $=500 \mu \mathrm{m}$.

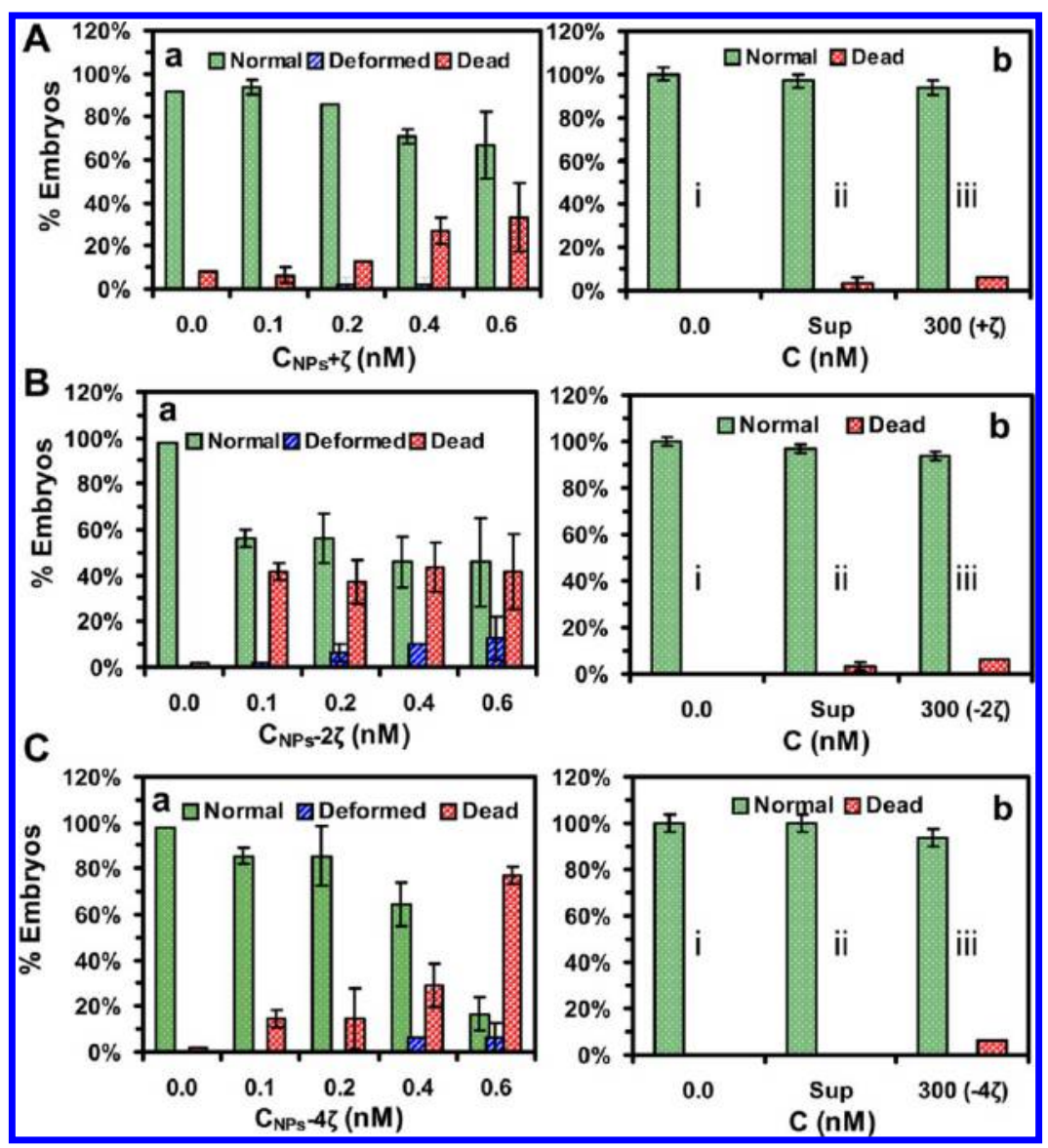

Figure 7. Study of dose-dependent and charge-dependent toxic effects of Ag-peptide NPs on embryonic developments. Histograms show the percentages of embryos that have been incubated with (a) the NPs: (A) Ag-CALNNK NPs ${ }^{+\zeta}$, (B) Ag-CALNNS NPs ${ }^{-2 \zeta}$, and (C) Ag-CALNNE $\mathrm{NPs}^{-4 \zeta}$; and (b) control experiments (absence of the NPs): (i) egg water, (ii) supernatant (collected from last washing of the NPs), and (iii) $300 \mathrm{nM}$ of the given peptide of (A) CALNNK, (B) CALNNS, or (C) CALNNE, for $120 \mathrm{~h}$, and develop to normal or deformed zebrafish, or become dead. 48 embryos are used for each NP concentration, and error bars represent standard deviations of 4 replicated experiments. 


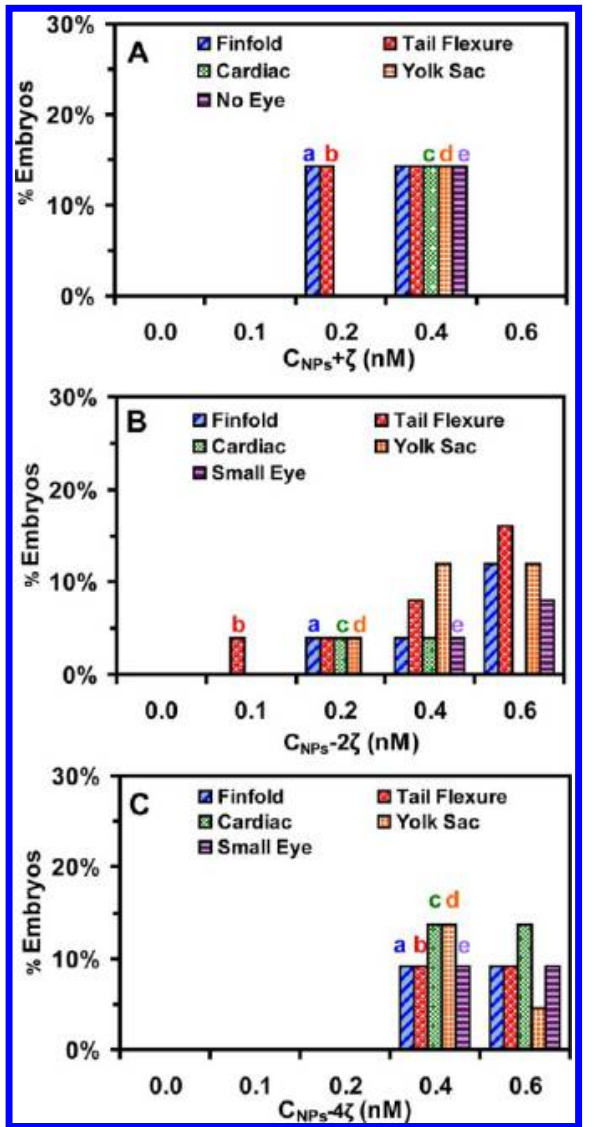

Figure 8. Study of dependence of types of deformities upon the doses and charges of Ag-peptide NPs. Histograms of distribution of percentages of embryos, which were incubated with the NPs (A) Ag-CALNNK NPs ${ }^{+\zeta}$, (B) Ag-CALNNS NPs ${ }^{-2 \zeta}$, and (C) Ag-CALNNE $\mathrm{NPs}^{-4 \zeta}$, for $120 \mathrm{~h}$ and developed to given types of deformities: (a) finfold abnormality; (b) tail/spinal cord flexure; (c) cardiac malformation; (d) yolk sac edema; (e) eye abnormality (no eye or small eye). The percentages of embryos that developed to each given deformity are calculated by dividing the number of embryos that developed to the given deformity by the total number of embryos that developed to all types of deformed zebrafish. Data represents the sum of all measurements. Thus, error bars are not applicable here.

Supporting Information) are $11.2 \pm 2.5,12.8 \pm 2.8$, and $11.9 \pm$ $2.8 \mathrm{~nm}$, which are similar to those measured using TEM (Figure 2). Molar absorptivity (extinction coefficient) of the Ag-peptide NPs is $2.6 \times 10^{8} \mathrm{M}^{-1} \mathrm{~cm}^{-1}$, which is smaller than the same-sized citrated Ag NPs $\left(9.8 \times 10^{8} \mathrm{M}^{-1} \mathrm{~cm}^{-1}\right){ }^{24,27}$ The result further indicates that the functionalization of Ag NPs with the peptides leads to the decrease of their surface reflectivity, dielectric constant, and absorptivity.

Real-Time Imaging of Charge-Dependent Transport of Single Ag-Peptide NPs into/in Embryos. To study charge-dependent diffusion and transport of single Ag-peptide NPs in cleavage-stage embryos, we incubated each given type of NPs with the embryos and tracked their diffusion into and in embryos in real time using DFOMS. We first determined how single Ag-peptide NPs enter into embryos from egg water (outside chorion, OC) and then their diffusion and transport inside the embryos. Optical images of the embryos (Figure 5A) show the chorionic layer (CL, the interface of egg water with chorionic space, CS), CS, and the interface of CS with inner mass of embryos (IME). The transport of the individual NPs through these interfaces into IME was imaged in vivo in real time at single NP resolution using DFOMS. The arrays of chorionic pore canals (CPCs) with diameters of $0.5-0.7 \mu \mathrm{m}$ and distances among neighboring pores at $1.5-2.5 \mu \mathrm{m}$ in the CL were directly imaged using DFOMS (Figure 5B-a and Figure S3-a in the Supporting Information), which agree well with those determined previously by $\mathrm{DFOMS}^{24-28}$ and by scanning electron microscopy (SEM). ${ }^{52}$

We found that all three Ag-peptide NPs diffused through chorionic pores to enter the CS, then diffused in the CS, and finally entered the IME, as shown in optical images and their diffusion trajectories (Figure 5B,C and Figures S3-S5 in the Supporting Information). We used the shortest exposure time (the highest speed) and monochrome (black/white) mode, instead of multichrome mode (color) of the CCD camera, to track diffusion of the single NPs in real time. We acquired the LSPR spectra (plasmonic colors) of the single NPs using colormode of the CCD camera (Figure 3) to distinguish them from debris and embryonic cells and membranes, which appeared white under dark-field illumination. We determined the sizes of single NPs in situ in real time using LSPR spectra of single Agpeptide NPs and the calibration approaches (optical nanoruler) as we reported previously. ${ }^{17,21,24,27}$ Single Ag-peptide NPs with similar LSPR spectra (sizes) were selected to minimize the potential effects of their sizes and to accurately study their diffusion modes and diffusion coefficients in various embryonic compartments.

We characterized the diffusion modes of each given type of NPs as they diffused into/in embryos by tracking diffusion trajectories of single NPs and plotting real-time square displacement (RTSD) versus diffusion time (Figure 5D and Figures $\mathrm{S} 3 \mathrm{c}-5 \mathrm{c}$ in the Supporting Information). We used RTSD (diffusion distance at each time interval) rather than mean-square displacement (MSD) (average distance over time) to determine diffusion modes of the single NPs, because embryonic environments are highly heterogeneous and change over time, as embryos develop.

Plots of RTSD versus time for each given type of single NPs that diffuse through CPCs into CS, in CS and IME (Figure 5D and Figures $\mathrm{S} 3 \mathrm{c}-5 \mathrm{c}$ in the Supporting Information) show stepwise linearity, suggesting highly heterogeneous embryonic environments. As described by diffusion theories, ${ }^{53-56}$ the linear plots indicate simple random Brownian motion and steps show restricted diffusion, showing that passive diffusion of single NPs (but not active cellular signaling) enables them to enter the embryos, and suggesting charge-independent diffusion modes of the Ag-peptide NPs. The results show constrained random Brownian motion for the NPs diffusing through CL into CS, and simple random Brownian motions for the NPs diffusing in CS and into/in IME. Individual NPs are trapped from time to time as they diffuse through the CL from egg water into CS and from CS to IME.

Interestingly, within the same duration of diffusion measurements and same embryonic environments (CL, CS, or IME), the positively charged Ag-CALNNK NPs ${ }^{+\zeta}$ diffuse the fastest and travel the longest distances, while the more negatively charged Ag-CALNNE $\mathrm{NPs}^{-4 \zeta}$ diffuse the slowest and the shortest distances (Figure 5D). Diffusion coefficients $(D)$ of single NPs are determined by dividing the slopes of linear portions of the plots with four using two-dimensional (2D) Random walk theory $(\mathrm{RTSD}=4 D \Delta t) .{ }^{55}$ (Note that each microscopic image is a $2 \mathrm{D}$ image, which acquires $2 \mathrm{D}$ diffusion of single NPs. Thus, 2D Random walk theory is used.) They are (i) $(1.7 \pm 0.4) \times 10^{-9}$, (ii) $(9.3 \pm 2.5) \times 10^{-10}$, and (iii) $(3.4 \pm$ 


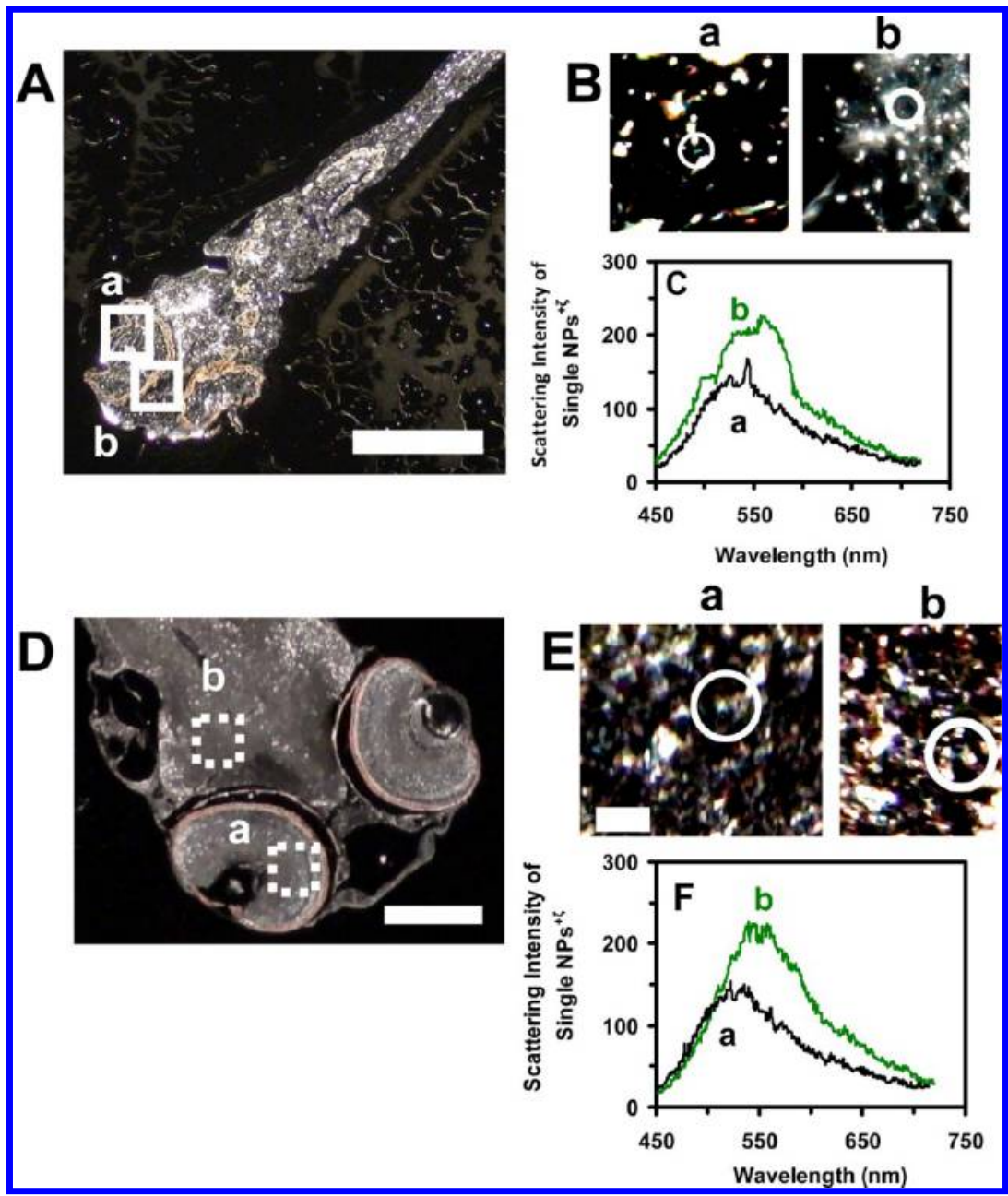

Figure 9. Study of individual Ag-CALNNK NPs ${ }^{+\zeta}$ embedded inside $(\mathrm{A}-\mathrm{C})$ deformed and (D-F) normally developed zebrafish using DFOMS. (A) Optical image of a fixed transverse section of a deformed zebrafish. The rectangles outline (a) eye and (b) brain tissues. (B) Zoom-in optical images of the tissues outlined in (A) show the NPs embedded in the tissues as circled. (C) Their LSPR spectra show plasmonic green NPs with $\lambda_{\max }$ (fwhm) (a) 545 (95) and (b) 559 (121) nm. (D) Optical image of a fixed transverse section of a normal zebrafish. The rectangles outline (a) eye and (b) brain tissues. (E) Zoom-in optical images of the tissue sections outlined in (D) show the NPs embedded in the tissues as circled. (F) Their LSPR spectra show plasmonic green NPs with $\lambda_{\max }$ (fwhm) (a) 532 (98) and (b) 551 (111) nm. Scale bars: (A) 250, (B) 50, (C) 500, and (D) $50 \mu \mathrm{m}$.

1.0) $\times 10^{-10} \mathrm{~cm}^{2} \mathrm{~s}^{-1}$ for the single green Ag-CALNNK NPs ${ }^{+\zeta}$, Ag-CALNNS NPs ${ }^{-2 \zeta}$, and Ag-CALNNE NPs ${ }^{-4 \zeta}$ going through CL from egg water into CS, respectively (Figure 5D-a). Their diffusion coefficients in CS (Figure 5D-b) are (i) $(4.6 \pm 0.9) \times$ $10^{-9}$, (ii) $(2.8 \pm 0.3) \times 10^{-9}$, and (iii) $(2.2 \pm 1.5) \times 10^{-9} \mathrm{~cm}^{2}$ $\mathrm{s}^{-1}$; and their diffusion coefficients in IME (Figure 5D-c) are (i) $(3.0 \pm 1.5) \times 10^{-9}$; (ii) $(1.5 \pm 0.4) \times 10^{-9}$, and (iii) $(4.6 \pm 0.7)$ $\times 10^{-10} \mathrm{~cm}^{2} \mathrm{~s}^{-1}$, respectively. The results show chargedependent diffusion coefficients, and suggest charge-dependent interactions of the NPs with the embryonic environments such that the positively charged Ag-CALNNK $\mathrm{NPs}^{+\zeta}$ have the weakest interactions with the embryonic environments, while the more negatively charged Ag-CALNNE $\mathrm{NPs}^{-4 \zeta}$ have the strongest interaction. Further studies are needed to determine their underlying molecular interactions.

Interestingly, diffusion coefficients of each type of Ag-peptide NPs (Ag-CALNNK NPs ${ }^{+\zeta}$, Ag-CALNNS NPs ${ }^{-2 \zeta}$, and AgCALNNE NPs $\left.{ }^{-4 \zeta}\right)$ in egg water are similar as $(4.4 \pm 1.1) \times$ $10^{-8},(4.9 \pm 1.2) \times 10^{-8}$, and $(5.6 \pm 2.0) \times 10^{-8}$, respectively. Note that diffusion coefficients of single NPs are inversely proportional to the sizes of NPs and viscosities of medium, as described by the Stokes-Einstein equation, $D=k T /(6 \pi \eta a)$, where $k$ is the Boltzmann constant; $T$ is temperature; $a$ is the radius of single NPs; and $\eta$ is viscosity of medium where NPs diffuse in. ${ }^{55}$ Thus, the results further show that the observed diffusion coefficients in Figure 5D are indeed attributed to their charge-dependent electrostatic interactions with embryonic molecules, but not their sizes. The results also show that embryonic environments are orders of magnitude more viscous than egg water, similar to those we reported previously, ${ }^{25-28}$ demonstrating that the Ag-peptide NPs can serve as effective optical imaging probes to study embryonic nanoenvironments.

Zebrafish Embryos as High-Throughput Ultrasensitive in Vivo Assays. Optical images in Figure 6A,B show normal developmental zebrafish embryos at cleavage (0.752.25 hours-post-fertilization, hpf), late segmentation ( $24 \mathrm{hpf})$, hatching (48 hpf), and protruding-mouth pharyngula stages (72 hpf), and fully developed larvae (120 hpf). Embryos are optically transparent, and their developmental stages are clearly defined, which enable them to be characterized simultaneously. Furthermore, a massive amount of embryos can be generated rapidly (overnight) at very low cost, which could serve as highthroughput in vivo assays to screen biocompatibility and toxicity of nanomaterials. 


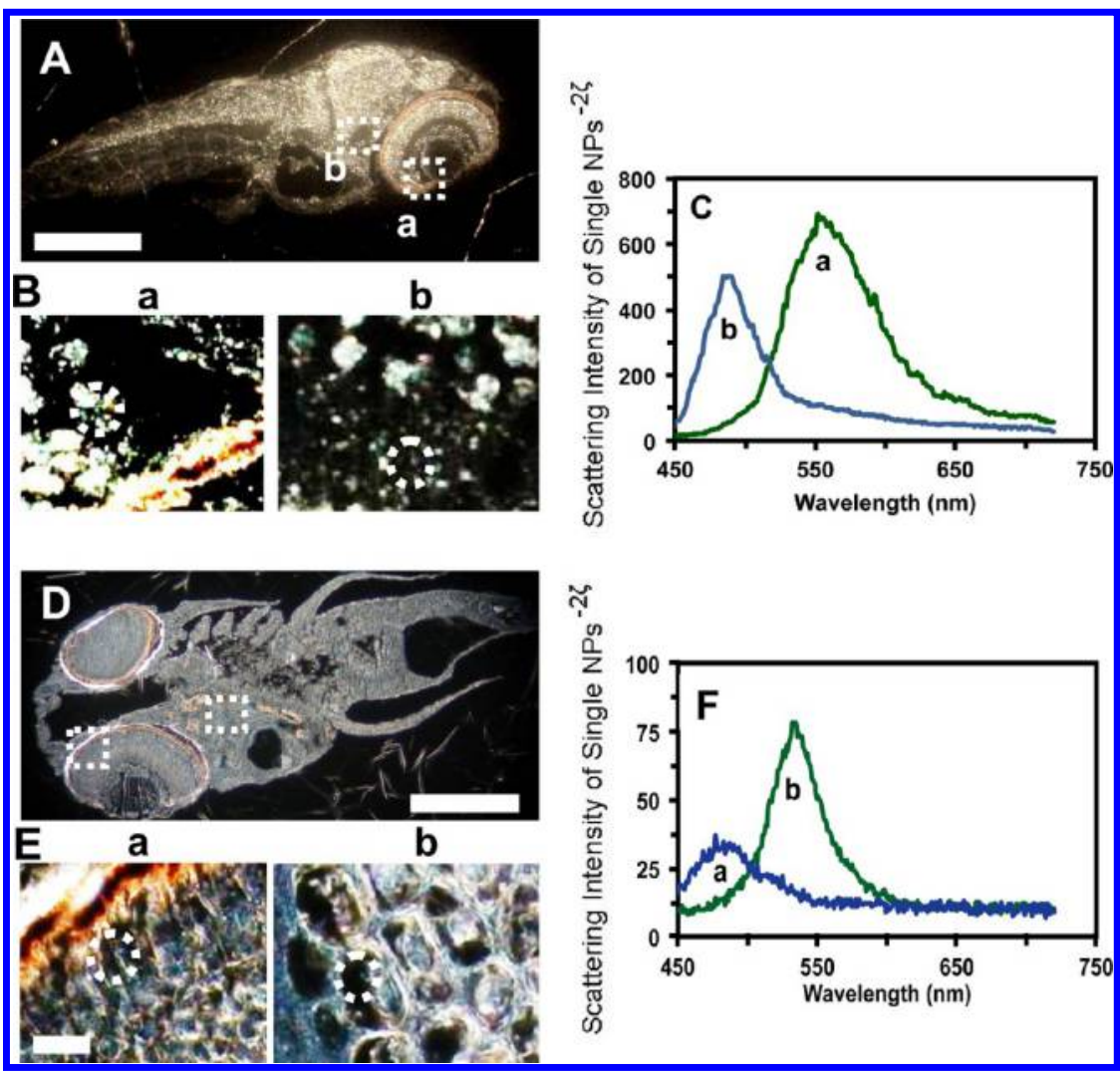

Figure 10. Characterization of individual Ag-CALNNS NPs ${ }^{-2 \zeta}$ embedded inside $(\mathrm{A}-\mathrm{C})$ deformed and (D-F) normally developed zebrafish using DFOMS. (A) Optical image of a fixed sagittal section of a deformed zebrafish. The rectangles outline (a) eye and (b) brain tissues. (B) Zoom-in optical images of the tissue sections outlined in (A) show the embedded NPs in the tissues as circled. (C) Their LSPR spectra show green NPs with $\lambda_{\max }($ fwhm) (a) 559 (111) and (b) 490 (99) nm. (D) Optical image of a fixed sagittal section of a normal zebrafish. The rectangles outline (a) eye and (b) brain tissues. (E) Zoom-in optical images of the tissue sections outlined in (D) show the embedded NPs in the tissues as circled. (F) Their LSPR spectra show green NPs with $\lambda_{\max }$ (fwhm) (a) 492 (101) and (b) 557 (112) nm. Scale bars: (A) 250, (B) 50, (C) 500, and (D) $50 \mu \mathrm{m}$.

Study of Dose and Charge-Dependent Biocompatibility and Toxicity of Ag-Peptide NPs. We studied dosedependent toxic effects of each given type of Ag-peptide NPs on embryonic development by incubating cleavage-stage embryos (Figure 6A) with various concentrations $(0-0.60$ $\mathrm{nM}$ or $0-3.43 \mu \mathrm{g} / \mathrm{mL}$ ) of the Ag-peptide NPs suspended in egg water for $120 \mathrm{~h}$. The cleavage-stage embryos undergo drastic changes and set forth the development of different organs for later embryonic developmental stages. ${ }^{41-43}$ Therefore, in this study, we select the cleavage-stage embryos to study charge- and dose-dependent transport, biocompatibility, and toxicity of the NPs, aiming to develop ultrasensitive in vivo assays to effectively characterize biocompatibility and toxicity of the NPs.

Molar concentrations $(0-0.60 \mathrm{nM})$ of the Ag NPs (but not $\mathrm{Ag}$ atom or peptides) were calculated and used to describe the dose- and charge-dependent biocompatibility and toxicity, as we reported previously. ${ }^{25-28,51}$ It is worth noting that weight and surface area of single NPs are proportional to the number (mole) of single NPs, and molar concentration of NPs accurately represents size, number (mole), and surface properties of the NPs. Therefore, the dose-dependent effects of NPs on embryonic development in molar concentrations represent dependence of nanotoxicity on their sizes, number, and surface properties. In contrast, the $\mathrm{w} / \mathrm{v}$ concentrations of NPs do not represent number (doses) of NPs, and cannot accurately reflect dependence of nanotoxicity on number and surface properties of the NPs, because the different-sized NPs with the same $\mathrm{w} / \mathrm{v}$ concentration contain different numbers of NPs, and thus different surface areas and charges of the NPs.

Representative embryonic developmental stages were imaged and assayed every $12 \mathrm{~h}$ over $120 \mathrm{hpf}$ until the embryos fully developed (Figure 6A,B). We determined and characterized the number of embryos that developed to normal zebrafish (Figure 6B), became dead (Figure 6C), or developed to deformed zebrafish (Figure 6D-F). The results in Figure 7 show various degrees of dependence of embryonic development upon the concentrations (doses) of all three Ag-peptide NPs. As the NP concentration increased from 0 to $0.6 \mathrm{nM}$, the number of embryos that developed to normal zebrafish decreased, while the number of embryos that became dead or developed to the deformed zebrafish increased.

All three Ag-peptide NPs generated several common and severely deformed zebrafish (Figure 6D-F), which included (a, b) finfold abnormality and tail and spinal cord flexure with nearly $180^{\circ}$ bend, (c) yolk sac edema with swollen and enlarged yolk sac, (d) cardiac malformations (edema of the pericardial sac region and cardiac arrhythmia), and (e) severe eye abnormalities with microphthalmia (small eyes) and dissymmetric eyes. The distributions of types of deformities versus the concentration of the Ag-peptide NPs in Figure 8 show that more embryos develop to severely deformed zebrafish (e.g., cardiac malformations and eye abnormalities) in the higher NP concentration.

Notably, we observe significant charge-dependent nanotoxicity among three Ag-peptide NPs and that the positively 


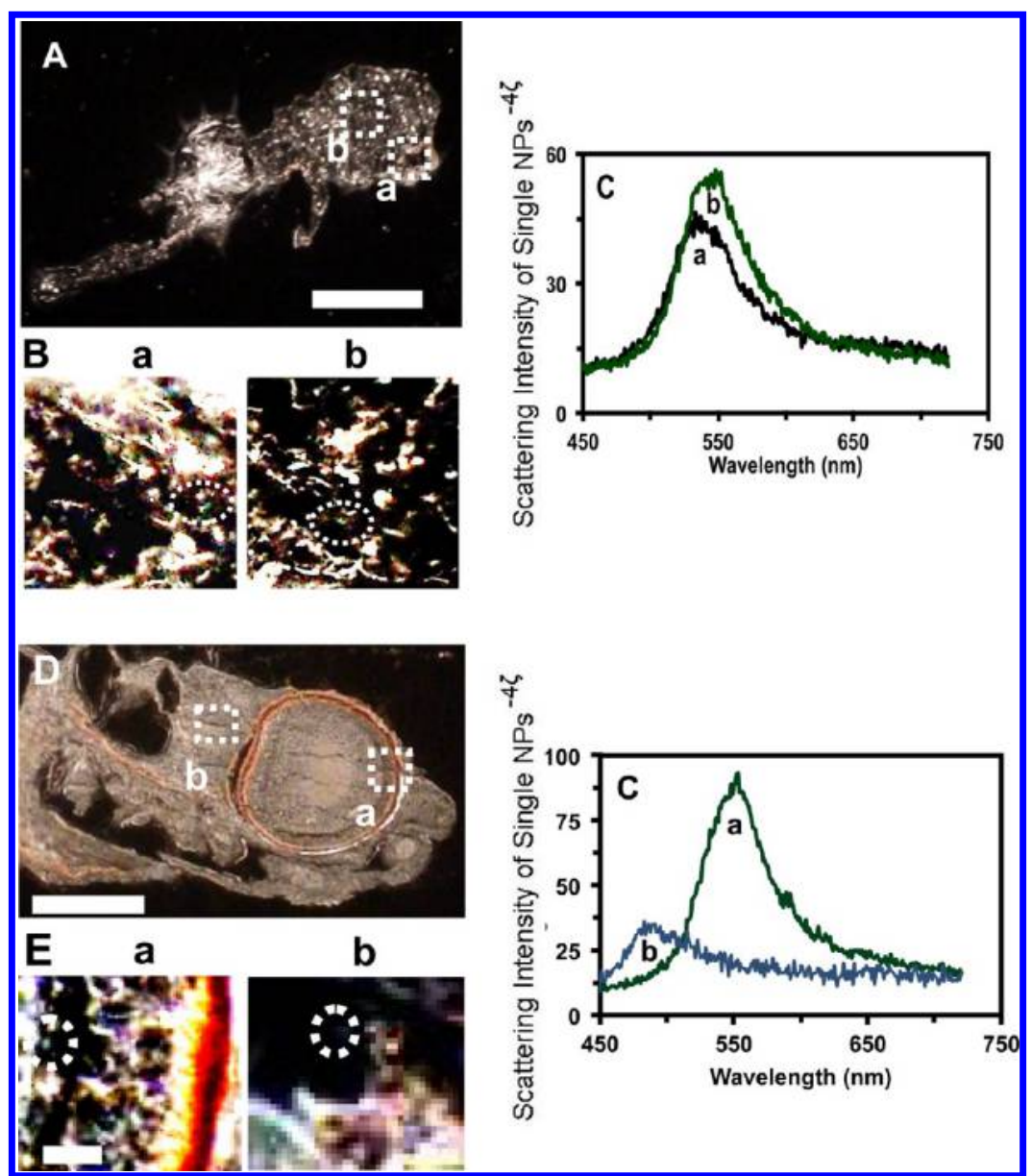

Figure 11. Characterization of individual Ag-CALNNE NPs ${ }^{-4 \zeta}$ embedded inside (A-C) deformed and (D-F) normally developed zebrafish using DFOMS. (A) Optical image of a fixed transverse section of a deformed zebrafish. The rectangles outline (a) eye and (b) brain tissues. (B) Zoom-in optical images of the tissue sections outlined in (A) show the embedded NPs in the tissues as circled. (C) Their LSPR spectra show green NPs with $\lambda_{\max }(\mathrm{fwhm})(\mathrm{a}) 548(102)$ and (b) 554 (121) nm. (D) Optical image of a fixed sagittal section of a normal zebrafish. The rectangles outline (a) eye and (b) brain tissues. (E) Zoom-in optical images of the tissue sections outlined in (D) show the embedded NPs in the tissues as circled. (F) Their LSPR spectra show green NPs with $\lambda_{\max }$ (fwhm) (a) 559 (109) and (b) 491 (101) nm. Scale bars: (A) 250, (B) 50, (C) 500, and (D) $50 \mu \mathrm{m}$.

charged Ag-CALNNK $\mathrm{NPs}^{+\zeta}$ are the most biocompatible with the embryonic development, while the more negatively charged Ag-CALNNE NPs ${ }^{-4 \zeta}$ create the highest toxicity toward the embryonic development (Figure 7A-a). For instance, the positively charged Ag-CALNNK $\mathrm{NPs}^{+\zeta}$ caused only $2 \%$ of embryos to become deformed zebrafish at 0.2 and $0.4 \mathrm{nM}$, and 13,27 , and $33 \%$ of the dead embryos for $0.2,0.4$, and $0.6 \mathrm{nM}$ NPs, respectively. The common types of deformed zebrafish (finfold and tail flexure) are observed at the lower NP concentration $(0.2 \mathrm{nM})$, and all five types of deformities are observed at the higher NP concentration (0.4 nM) (Figure 8A).

In contrast, the more negatively charged Ag-CALNNE $\mathrm{NPs}^{-4 \zeta}$ caused $6 \%$ of embryos to become deformed zebrafish with all five types of deformities at both 0.4 and $0.6 \mathrm{nM}$, and $15 \%$ of the dead embryos for the NP concentration as low as 0.1 and $0.2 \mathrm{nM}$, and $29 \%$ and $77 \%$ of the dead at 0.4 and 0.6 $\mathrm{nM}$, respectively (Figure 7B-a). The low negatively charged AgCALNNS $\mathrm{NPs}^{-2 \zeta}$ also created dose-dependent deformed zebrafish, 2, 6, 10, and $13 \%$ with finfold abnormality for 0.1 $\mathrm{nM}$, and all other four and five deformities for $0.2,0.4$, and 0.6 $\mathrm{nM}$, respectively (Figures 7C-a and $8 \mathrm{C}$ ). Furthermore, the AgCALNNS $\mathrm{NPs}^{-2 \zeta}$ caused the highest percentage of dead embryos $(42 \pm 10) \%$ for NP concentration as low as $0.1 \mathrm{nM}$, which remained essentially uncharged as its concentration increased to $0.2,0.4$, and $0.6 \mathrm{nM}$.

Three control experiments were conducted simultaneously by incubating the embryos with egg water alone (blank control), the highest concentrations of supernatants collected from the last washing of the given Ag-peptide NPs, and given peptides (Figure $7 \mathrm{~b}$ ). The results show that over $95 \%$ embryos developed to normal zebrafish, and none of the embryos developed to deformed zebrafish, indicating that the observed toxic effects of the NPs on embryonic development (Figures 6-8) are attributed to the NPs, but not other chemicals, and the peptides are biocompatible with embryonic development. Notably, if the Ag NPs released any sufficient amount of silver cation $\left(\mathrm{Ag}^{+}\right)$that caused significant toxic effects on embryonic development, we would have observed them in the control experiments with supernatants. Furthermore, the positively charged Ag-peptide NPs would have released more $\mathrm{Ag}^{+}$because of their same charge repulsion, and caused higher toxicity than the negatively charged NPs. However, that was not what we observed. Thus, the control experiments with the supernatants enable us to eliminate potential toxic effects of trace chemicals (e.g., $\mathrm{Ag}^{+}$) resulting from $\mathrm{Ag}$ NP synthesis or their degradation over time. 
Taken together, these interesting findings offer reliable new evidence, which shows charge-dependent toxic effects of Agpeptide NPs on embryonic development in vivo in real time. This study shows that the positively charged Ag-CALNNK $\mathrm{NPs}^{+\zeta}$ with zeta potential of $(3.0 \pm 0.2) \mathrm{mV}$ are more biocompatible than both negatively charged Ag-CALNNS $\mathrm{NPs}^{-2 \zeta}$ and Ag-CALNNE NPs ${ }^{-4 \zeta}$. The lower negatively charged Ag-CALNNS $\mathrm{NPs}^{-2 \zeta}$ with zeta potential of $-(5.0 \pm$ $0.1) \mathrm{mV}$ are more biocompatible than the higher negatively charged Ag-CALNNE NPs ${ }^{-4 \zeta}$ with zeta potential of $-(11.9 \pm$ 0.9) $\mathrm{mV}$. The results suggest that electrostatic interactions of the charged NPs with embryonic molecules may be responsible for the observed charge-dependent nanotoxicity. However, physicochemical properties of intraembryonic environments remain largely unknown. Thus, further studies are needed to characterize underlying molecular mechanisms of the chargedependent effects of the NPs.

Quantitative Analysis of Single Ag-Peptide NPs Embedded in Deformed and Normal Zebrafish. To determine why some embryos developed normally and others became deformed or dead, we incubated cleavage-stage embryos with each given type of Ag-peptide NPs (0-0.6 $\mathrm{nM}$ ) chronically (continuously) for $120 \mathrm{~h}$ until they developed to normal and deformed zebrafish. We prepared thin-layer sections (1-2 $\mu \mathrm{m}$ thickness) of the eye and brain tissues of normal and deformed zebrafish, and quantitatively imaged and characterized single Ag-peptide NPs embedded in the tissues. We are especially interested in probing whether and how the NPs cause severe abnormality of the eyes and brains, which are biomedically important organs.

The tissue sections $(1-2 \mu \mathrm{m}$ thickness) of representative deformed zebrafish with all types of deformities show the NPs embedded in its eye (retina) and brain (Figure 9A-C), as characterized by distinctive LSPR spectra of individual AgCALNNK NPs ${ }^{+\zeta}$. Interestingly, the tissue sections of normally developed zebrafish (Figure 9D-F) also show the NPs embedded in the tissues, similar to those of deformed zebrafish. The results show that the positively charged Ag-peptide NPs diffuse into embryos and stay inside the embryos throughout their entire development (120 hpf). Similar phenomena are observed for both Ag-CALNNS $\mathrm{NPs}^{-2 \zeta}$ and Ag-CALNNE $\mathrm{NPs}^{-4 \zeta}$ (Figures 10 and 11).

The number of each given type of Ag-peptide NPs embedded in eye (retina) and brain tissues of normal and deformed zebrafish (Figure 12) shows that more NPs are present in deformed zebrafish than normal ones, indicating that the accumulation of a higher amount of NPs in developing embryos throughout their development $(120 \mathrm{~h})$ might have caused their deformation. Notably, we observe the nearly identical number of each type of NPs in the same detection volumes of the same type of tissues of either deformed or normal zebrafish, respectively. The results further demonstrate that all three types of Ag-peptide NPs diffuse into the embryos and stay inside the embryos throughout their development $(120 \mathrm{~h})$, and their different toxic effects on embryonic development (Figures 7 and 8) are attributed to their charges, but not their accumulated doses.

Unlike what we observed in our previous studies, ${ }^{25-28}$ plasmonic green NPs (but not other colors) are found in the tissues of both deformed and normal zebrafish. Their LSPR spectra show plasmonic green colored NPs with $\lambda_{\max }$ (fwhm), ranging from 490 (99) to 559 (111) nm (Figures 9-11), which are the same as those used to incubate with the embryos

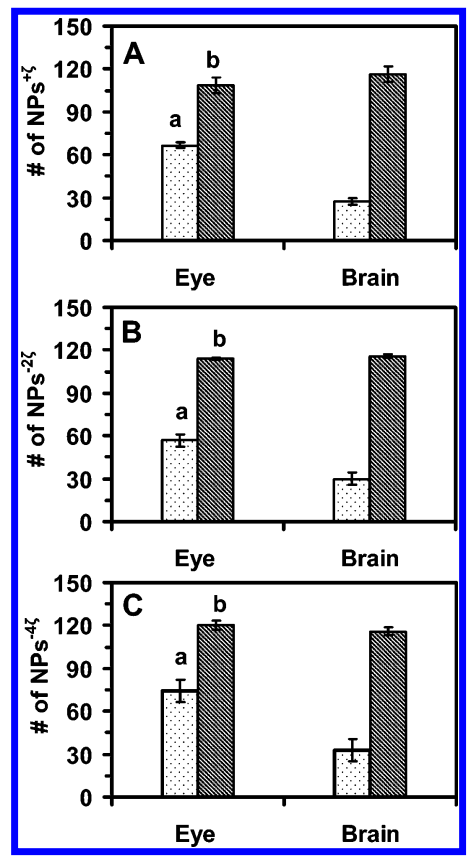

Figure 12. Quantitative analysis of number of single Ag-peptide NPs embedded in the eye and brain tissues of (a) normal and (b) deformed zebrafish: (A) Ag-CALNNK NPs ${ }^{+\zeta}$, (B) Ag-CALNNS $\mathrm{NPs}^{-2 \zeta}$, and (C) Ag-CALNNE $\mathrm{NPs}^{-4 \zeta}$, shows that more NPs are accumulated in the tissues of deformed zebrafish than normally developed zebrafish.

(Figure 3). The results demonstrate that the NPs remain unchanged over their $120 \mathrm{~h}$ incubation with the embryos, showing that the Ag-peptide NPs are much more stable (nonaggregated) in the tissues than the citrated Ag NPs. The result suggests the higher affinity of - $\mathrm{SH}$ with the surface of Ag NPs than the electrostatic interactions of citrates with the surfaces, which can be highly altered by the $\mathrm{pH}$ and ionic strength of surrounding medium.

It is worth noting that single NP plasmonic spectroscopy and DFOMS enable us to achieve such quantitative analysis of individual Ag-peptide NPs embedded in the tissues with submicrometer $(\mu \mathrm{m})$ spatial resolution and $100 \mathrm{~ms}$ temporal resolution. Even though it is hard to visualize single NPs in the images (Figures 9-11: B and E), the distinctive LSPR spectra of single Ag-peptide NPs (Figures 9-11: C and F), subtracted from the tissue background, enable us to unambiguously detect and quantitatively image single Ag-peptide NPs embedded in the tissues, which has not yet been achieved by other optical imaging methods. The analysis of single NPs using DFOMS is rapid, which offers high-throughput capabilities. Furthermore, imaging of single NPs using DFOMS is non-destructive, which preserves the tissues and enable them to be repeatedly characterized using multiple detection methods.

Note that each deformed zebrafish is distinctive and shows specific phenotypes (none of them identical), and the tissues of eyes and brains of each deformed zebrafish are very small $(\mu \mathrm{m}$ sizes), underscoring the importance of analysis of embedded single NPs in individual tissues of interest. The conventional ensemble analytical methods (e.g., inductively coupled plasma mass spectrometry or atomic absorption spectroscopy) are unsuited for such measurements, because one microsized tissue would have to be surgically removed, dried, and weighed (to ensure the same amount of each sample to be analyzed), dissolved by acids to convert the $\mathrm{Ag}$ NPs to $\mathrm{Ag}^{+}$, and then analyzed. These conventional analysis tools neither offer any 
spatial resolution nor provide sufficient sensitivity to quantitatively analyze single NPs embedded in tiny tissues. They are also time-consuming and destructive, which completely destroys the tissue samples and prevents them from being analyzed using various means.

New Findings of This Study. Several other studies have reported charge-dependent cytotoxicity of nanomaterials. ${ }^{57-59}$ However, the studies are inconclusive. Majority studies showed that positively charged NPs were more toxic toward given types of cell lines than negatively charged NPs, and assumed that the interactions of positively charged NPs with the cell membrane caused the toxicity. Unlike this study, these previous studies used in vitro cell assays and studied cytotoxicity, which has numerous limitations (e.g., cell-line dependence). Furthermore, these previous studies used highly charged NPs with zeta potentials of -50 or $+50 \mathrm{mV}$, and conventional chemicals as surface functional groups, which could be toxic themselves.

Many of these previous studies did not use purified and stable NPs, and did not perform control experiments with the surface functional molecules. Consequently, it is unclear whether those observed toxicities are attributed to the charges of the NPs, aggregation of the NPs, residual chemicals, or their surface functional molecules. Notably, all previous studies were not carried out at single-NP resolution, but with conventional ensemble measurements, which could not characterize dose (number) and size of single NPs in situ in real time. Thus, it is unclear whether those observed toxicities are attributed to their various doses, sizes, or charges.

In our previous studies, ${ }^{25,27}$ we synthesized, purified, and characterized same-sized citrated Ag NPs $(11.6 \pm 3.5 \mathrm{~nm})$ and $\mathrm{Au}$ NPs $(11.6 \pm 0.9 \mathrm{~nm})$ with zeta potential of $-5 \mathrm{mV}$ in egg water. We used the identical experimental approaches and conditions as those described in this study, to determine their effects on embryonic developments. The results show that the Ag NPs generate toxic effects on embryonic development in a dose-dependent manner. In contrast, the same-sized Au NPs are much more biocompatible with embryonic development. These previous studies clearly show the chemical compositiondependent toxic effects of $\mathrm{Ag}$ and $\mathrm{Au} \mathrm{NPs}$ on embryonic development.

We also used the same approaches to study larger Ag NPs $(41.6 \pm 9.1$ and $95.4 \pm 16.0 \mathrm{~nm})$ and Au NPs $(86.2 \pm 10.8$ $\mathrm{nm}){ }^{26,28}$ The results show that larger Ag NPs create higher toxic effects on embryonic development than smaller Ag NPs. In contrast, the Au NPs display size-independent toxicity, and larger Au NPs $(86.2 \pm 10.8 \mathrm{~nm})$ are as biocompatible as smaller $\mathrm{Au}$ NPs $(11.6 \pm 0.9 \mathrm{~nm})$.

In this study, the results show that the Ag-peptide NPs share the same diffusion modes as the same-sized citrated $\mathrm{Ag}$ and $\mathrm{Au}$ NPs. Notably, all three types of Ag-peptide NPs are more biocompatible with embryonic development than the samesized citrated Ag NPs. Among three Ag-peptide NPs, the positively charged Ag-CALNNK $\mathrm{NPs}^{+\zeta}$ are the most biocompatible, which are nearly as biocompatible as the same-sized citrated Au NPs. In contrast, the highly negative charged AgCALNNE NPs ${ }^{-4 \zeta}$ are the most toxic ones, but still much more biocompatible than the citrated Ag NPs. Interestingly, the AgCALNNS $\mathrm{NPs}^{-2 \zeta}$ with the nearly identical zeta potential as those of the citrated Ag NPs $(-5 \mathrm{mV})$ create less toxic effects on embryonic development than the citrated Ag NPs.

Taken together, the results show the dependence of biocompatibility and toxicity of the NPs upon their surface charges, surface functional groups (peptide or citrates), and chemical compositions (Ag or Au NPs). Our studies show that toxic effects of the NPs on embryonic development are defined by the combination of multiple physicochemical properties of the NPs, underscoring the importance of probing their effects one factor at a time. It is worth noting that these distinctive findings cannot be extrapolated from any previous studies, and cannot be generally applied to other types or other charges of NPs.

\section{SUMMARY}

In summary, we have synthesized, purified, and characterized three types of Ag-peptide NPs with nearly spherical shapes and average diameters of $(11.7 \pm 2.7) \mathrm{nm}$. They are either positively or negatively charged with zeta potentials of $(3.0 \pm$ $0.2),-(5.0 \pm 0.1)$, or $-(11.9 \pm 0.9) \mathrm{mV}$. We further developed our DFOMS for real-time quantitative imaging of single Agpeptide NPs in vivo in situ in real time, and in the tissues of interest with both spatial and temporal resolutions. The results show that all three Ag-peptide NPs are very stable (nonaggregated) in egg water, embryos, and zebrafish over the entire duration of embryonic development (120 hpf). They passively diffuse into embryos via embryonic chorionic pores, and stay inside the embryos throughout the embryonic development (120 hpf), showing charge-independent diffusion modes and charge-dependent diffusion coefficients. The results show distinctive charge-dependent toxic effects on the embryonic development, that the positively charged Ag-CALNNK NPs ${ }^{+\zeta}$ create the lowest toxic effects, while the highly negatively charged Ag-CALNNE NPs ${ }^{-4 \zeta}$ cause the highest toxic effects on the embryonic development. By comparing with our previous studies of same-sized negatively charged citrated $\mathrm{Ag}$ and $\mathrm{Au}$ NPs with zeta potential of $-5 \mathrm{mV},{ }^{25,27}$ all three Ag-peptide NPs are far less toxic than the citrated Ag NPs. The Ag-CALNNK $\mathrm{NPs}^{+\zeta}$ are nearly as biocompatible as the Au NPs, while both negatively charged Ag-peptide NPs are slightly more toxic than the $\mathrm{Au}$ NPs. Taken together, the results show the dependence of toxic effects of NPs toward embryonic development upon their surface charges, surface functional groups (peptide, citrate), and chemical compositions ( $\mathrm{Ag}, \mathrm{Au})$, and suggest the possibility of rational design of biocompatible NPs for a wide variety of applications. This study shows that the embryos can serve as ultrasensitive in vivo assays for study of surface chargedependent toxicity of NPs. The study also demonstrates the powerful applications of DFOMS for real-time quantitative study of the transport and effects of NPs on embryonic development, and for quantitative analysis of single NPs embedded in the tissues.

\section{ASSOCIATED CONTENT}

\section{S Supporting Information}

Characterization of stability of Ag-CALNNS $\mathrm{NPs}^{-2 \zeta}$ (Figure S1) and Ag-CALNNE NPs ${ }^{-4 \zeta}$ (Figure S2) in egg water. Realtime study of charge-dependent transport and diffusion of single NPs in CL (Figure S3), in CS (Figure S4), and in IME (Figure S5). This material is available free of charge via the Internet at http://pubs.acs.org.

\section{AUTHOR INFORMATION}

\section{Corresponding Author}

*E-mail: xhxu@odu.edu. URI: www.odu.edu/sci/xu/xu.htm. Tel/fax: (757) 683-5698. 


\section{Funding}

This work is supported in part by NSF (NIRT: CBET 0507036) and NIH (R01 GM076440; 3R01 GM076440-04S1). K.J.L., P.K.N., and L.M.B. are grateful for the support of NSFGRAS (CBET 1042533), Dominion Scholar Fellowship, and NIH-GRAS (R01 3GM0764401-S1), respectively.

\section{Notes}

The authors declare no competing financial interest.

\section{ABBREVIATIONS}

$\mathrm{Ag}$, silver; $\mathrm{Au}$, gold; $\mathrm{CL}$, chorionic layer; $\mathrm{CS}$, chorionic space; CPC, chorionic pore canals; DFOMS, dark-field optical microscopy and spectroscopy; DI, deionized; DLS, dynamic light scattering; fwhm, full-width-at-half-maximum; hpf, hourspost-fertilization; HRTEM, high-resolution transmission electron microscope; IME, inner mass of embryo; LSPR, localized surface plasmon resonance; MSD, mean square displacement; MSIS, multispectral imaging system; nm, nanometer; NP, nanoparticle; OC, outside chorion; PEG, polyethylene glycol; RTSD, real-time square displacement; w/v, weight/volume

\section{REFERENCES}

(1) Ahamed, M., Alsalhi, M. S., and Siddiqui, M. K. (2010) Silver nanoparticle applications and human health. Clin. Chim. Acta 411, 1841-1848.

(2) Binsalamah, Z. M., Paul, A., Prakash, S., and Shum-Tim, D. (2012) Nanomedicine in cardiovascular therapy: recent advancements. Expert Rev. Cardiovasc. Ther. 10, 805-815.

(3) Chaloupka, K., Malam, Y., and Seifalian, A. M. (2010) Nanosilver as a new generation of nanoproduct in biomedical applications. Trends Biotechnol. 28, 580-588.

(4) Saracino, G. A., Cigognini, D., Silva, D., Caprini, A., and Gelain, F. (2012) Nanomaterials design and tests for neural tissue engineering. Chem. Soc. Rev. 42, 225-262.

(5) Teli, M. K., Mutalik, S., and Rajanikant, G. K. (2010) Nanotechnology and nanomedicine: going small means aiming big. Curr. Pharm Des. 16, 1882-1892.

(6) Xu, X.-H. N., and Patel, R. P. (2005) Imaging and Assembly of Nanoparticles in Biological Systems, in Handbook of Nanostructured Biomaterials and Their Applications in Nanobiotechnology (Nalwa, H. S., Ed.) pp 435-456, American Scientific Publishers, Los Angeles, CA.

(7) Huang, T., Nallathamby, P. D., and Xu, X.-H. N. (2008) Photostable single-molecule nanoparticle optical biosensors for realtime sensing of single cytokine molecules and their binding reactions. J. Am. Chem. Soc. 130, 17095-17105.

(8) Nallathamby, P. D., and Xu, X.-H. N. (2010) Study of cytotoxic and therapeutic effects of stable and purified silver nanoparticles on tumor cells. Nanoscale 2, 942-952.

(9) Huang, T., Nallathamby, P. D., Gillet, D., and Xu, X.-H. N. (2007) Design and synthesis of single nanoparticle optical biosensors for imaging and characterization of single receptor molecules on single living cells. Anal. Chem. 79, 7708-7718.

(10) Huang, T., and Xu, X.-H. N. (2011) Multicolored nanometerresolution mapping of single protein-ligand binding complex using farfield photostable optical nanoscopy (PHOTON). Nanoscale 3, 35673572.

(11) Haes, A. J., and Van Duyne, R. P. (2002) A nanoscale optical biosensor: sensitivity and selectivity of an approach based on the localized surface plasmon resonance spectroscopy of triangular silver nanoparticles. J. Am. Chem. Soc. 124, 10596-10604.

(12) Huang, T., Browning, L. M., and Xu, X.-H. N. (2012) Far-field photostable optical nanoscopy (PHOTON) for real-time superresolution single-molecular imaging of signaling pathways of single live cells. Nanoscale 4, 2797-2812.

(13) Bohren, C. F., and Huffman, D. R. (1983) in Absorption and Scattering of Light by Small Particles, pp 287-380, Wiley, New York.
(14) Kelly, K. L., Coronado, E., Zhao, L. L., and Schatz, G. C. (2003) The optical properties of metal nanoparticles: the influence of size, shape, and dielectric environment. J. Phys. Chem. B 107, 668-677.

(15) Kreibig, U., and Vollmer, M. (1995) in Optical Properties of Metal Clusters, pp 14-123, Springer, Berlin.

(16) Mie, G. (1908) Beiträg zur optik trüber medien, speziell kolloidaler metrallősungen. Annu. Phys. 25, 377-445.

(17) Nallathamby, P. D., Huang, T., and Xu, X.-H. N. (2010) Design and characterization of optical nano rulers of single nanoparticles using optical microscopy and spectroscopy. Nanoscale 2, 1715-1722.

(18) Huang, T., and Xu, X.-H. N. (2010) Synthesis and characterization of tunable rainbow colored silver nanoparticle solutions using single-nanoparticle plasmonic microscopy and spectroscopy. J. Mater. Chem. 20, 9867-9876.

(19) Mulvaney, P. (1996) Surface plasmon spectroscopy of nanosized metal particles. Langmuir 12, 788-800.

(20) Lee, K. J., Browning, L. M., Huang, T., Ding, F., Nallathamby, P. D., and Xu, X.-H. N. (2010) Probing of multidrug ABC membrane transporters of single living cells using single plasmonic nanoparticle optical probes. Anal. Bioanal. Chem. 397, 3317-3328.

(21) Nallathamby, P. D., Lee, K. J., Desai, T., and Xu, X.-H. N. (2010) Study of multidrug membrane transporters of single living pseudomonas aeruginosa cells using size-dependent plasmonic nanoparticle optical probes. Biochemistry 49, 5942-5953.

(22) Xu, X.-H. N., Brownlow, W. J., Kyriacou, S. V., Wan, Q., and Viola, J. J. (2004) Real-time probing of membrane transport in living microbial cells using single nanoparticle optics and living cell imaging. Biochemistry 43, 10400-10413.

(23) Xu, X.-H. N., Chen, J., Jeffers, R. B., and Kyriacou, S. V. (2002) Direct measurement of sizes and dynamics of single living membrane transporters using nano-optics. Nano Lett. 2, 175-182.

(24) Nallathamby, P. D., Lee, K. J., and Xu, X.-H. N. (2008) Design of stable and uniform single nanoparticle photonics for in vivo dynamics imaging of nanoenvironments of zebrafish embryonic fluids. ACS Nano 2, 1371-1380.

(25) Browning, L. M., Lee, K. J., Huang, T., Nallathamby, P. D., Lowman, J., and $\mathrm{Xu}, \mathrm{X} .-\mathrm{H}$. N. (2009) Random walk of single gold nanoparticles in zebrafish embryos leading to stochastic toxic effects on embryonic developments. Nanoscale 1, 138-152.

(26) Lee, K. J., Browning, L. M., Nallathamby, P. D., Desai, T., Cherukui, P., and Xu, X.-H. N. (2012) In vivo quantitative study of size-dependent transport and toxicity of single silver nanoparticles using zebrafish embryos. Chem. Res. Toxicol. 25, 1029-1046.

(27) Lee, K. J., Nallathamby, P. D., Browning, L. M., Osgood, C. J., and $\mathrm{Xu}, \mathrm{X} . \mathrm{H}$. N. (2007) In vivo imaging of transport and biocompatibility of single silver nanoparticles in early development of zebrafish embryos. ACS Nano 1, 133-143.

(28) Lee, K. J., Nallathamby, P. D., Browning, L. M., Desai, T., Cherukui, P., and $\mathrm{Xu}, \mathrm{X} . \mathrm{H}$. N. (2012) Single nanoparticle spectroscopy for real-time in vivo quantitative analysis of transport and toxicity of single nanoparticles in single embryos. Analyst 137, 2973-2986.

(29) Browning, L. M., Huang, T., and Xu, X.-H. N. (2013) Real-time in vivo imaging of size-dependent transport and toxicity of gold nanoparticles in zebrafish embryos using single nanoparticle plasmonic spectroscopy, Interface Focus, 3, 20120098.

(30) Fabrega, J., Fawcett, S. R., Renshaw, J. C., and Lead, J. R. (2009) Silver nanoparticle impact on bacterial growth: effect of $\mathrm{pH}$, concentration, and organic matter. Environ. Sci. Technol. 43, 72857290.

(31) Hillegass, J. M., Shukla, A., Lathrop, S. A., MacPherson, M. B., Fukagawa, N. K., and Mossman, B. T. (2010) Assessing nanotoxicity in cells in vitro. Wiley Interdiscip. Rev. Nanomed. Nanobiotechnol. 2, 219231.

(32) Buzea, C., Pacheco, I. I., and Robbie, K. (2007) Nanomaterials and nanoparticles: sources and toxicity. Biointerphases 2, MR17-71.

(33) Nel, A. E., Madler, L., Velegol, D., Xia, T., Hoek, E. M., Somasundaran, P., Klaessig, F., Castranova, V., and Thompson, M. (2009) Understanding biophysicochemical interactions at the nanobio interface. Nat. Mater. 8, 543-557. 
(34) Bhattacharya, R., and Mukherjee, P. (2008) Biological properties of "naked" metal nanoparticles. Adv. Drug Delivery Rev. 60, 12891306.

(35) Khlebtsov, N., and Dykman, L. (2011) Biodistribution and toxicity of engineered gold nanoparticles: a review of in vitro and in vivo studies. Chem. Soc. Rev. 40, 1647-1671.

(36) Fadeel, B., and Garcia-Bennett, A. E. (2010) Better safe than sorry: understanding the toxicological properties of inorganic nanoparticles manufactured for biomedical applications. Adv. Drug Delivery Rev. 62, 362-374.

(37) Fischer, H. C., and Chan, W. C. (2007) Nanotoxicity: The growing need for in vivo study. Curr. Opin. Biotechnol. 18, 565-571.

(38) AshaRani, P. V., Mun, G. L., Hande, M. P., and Valiyaveettil, S. (2009) Cytotoxicity and genotoxicity of silver nanoparticles in human cells. ACS Nano 3, 279-290.

(39) Bar-Ilan, O., Albrecht, R. M., Fako, V. E., and Furgeson, D. Y. (2009) Toxicity assessments of multisized gold and silver nanoparticles in zebrafish embryos. Small 5, 1897-1910.

(40) Shaw, B. J., and Handy, R. D. (2011) Physiological effects of nanoparticles on fish: a comparison of nanometals versus metal ions. Environ. Int. 37, 1083-1097.

(41) den Hertog, J. (2005) Chemical Genetics: Drug Screens In Zebrafish. Biosci. Rep. 25, 289-297.

(42) Hill, A. J., Teraoka, H., Heideman, W., and Peterson, R. E. (2005) Zebrafish as a model vertebrate for investigating chemical toxicity. Toxicol. Sci. 86, 6-19.

(43) Kahn, P. (1994) Zebrafish hit the big time. Science 264, 904905.

(44) Lee, P. C., and Meisel, D. (1982) Adsorption and surfaceenhanced Raman of dyes on silver and gold sols. J. Phys. Chem. 86, 3391-3395.

(45) Doty, R. C., Tshikhudo, T. R., Brust, M., and Fernig, D. G. (2005) Extremely stable water-soluble Ag nanoparticles. Chem. Mater. 17, 4630-4635.

(46) Wang, Z., Levy, R., Fernig, D. G., and Brust, M. (2005) The peptide route to multifunctional gold nanoparticles. Bioconjugate Chem. 16, 497-500.

(47) Kyriacou, S. V., Brownlow, W. J., and Xu, X. H. N. (2004) Using nanoparticle optics assay for direct observation of the function of antimicrobial agents in single live bacterial cells. Biochemistry 43, 140147.

(48) Gebhart, S. C., Thompson, R. C., and Mahadevan-Jansen, A. (2007) Liquid-crystal tunable filter spectral imaging for brain tumor demarcation. Appl. Opt. 46, 1896-1910.

(49) Huang, T., Cai, W., Elsayed-Ali, H. E., and Xu, X. H. N. (2012) High-throughput ultrasensitive characterization of chemical, structural and plasmonic properties of EBL-fabricated single silver nanoparticles. Nanoscale 4, 380-385.

(50) Westerfield, M. (Chapters 1-4, 1993 (http://zfin.org/zf_info/ zfbook/zfbk.html)) The zebrafish book: A Guide for the Laboratory Use of Zebrafish (Danio Rerio*), University of Oregon Press Eugene, OR.

(51) Xu, X.-H. N., Huang, S., Brownlow, W., Salatia, K., and Jeffers, R. (2004) Size and temperature dependence of surface plasmon absorption of gold nanoparticles induced by tris $\left(2,2^{\prime}\right.$-bipyridine $)$ ruthenium(II). J. Phys. Chem. B 108, 15543-15551.

(52) Rawson, D. M., Zhang, T., Kalicharan, D., and Jongebloed, W. L. (2000) Field emission scanning electron microscopy and transmission electron microscopy studies of the chorion, plasma membrane and syncytial layers of the gastrula stage embryo of the zebrafish brachydanio rerio: a consideration of the structural and functional relationships with respect to cryoprotectant penetration. Aquacult. Res. $31,325-336$.

(53) Kusumi, A., and Sako, Y. (1994) Compartmental structure of the plasma membrane for receptor movements as revealed by a nanometer-level motion analysis. J. Cell Biol. 125, 1251-1264.

(54) Kusumi, A., Sako, Y., and Yamamoto, M. (1993) Confined lateral diffusion of membrane receptors as studied by single particle tracking (nanovid microscopy). effects of calcium-induced differentiation in cultured epithelial cells. Biophys. J. 65, 2021-2040.
(55) Tinoco, I., Sauer, K., Wang, J., and Puglisi, J. D. (2002) Molecular Motion and Transport Properties, in Physical ChemistryPrinciples and Applications in Biological Sciences, pp 274-290, Prentice Hall, NJ.

(56) Qian, H., Sheetz, M. P., and Elson, E. L. (1991) Single particle tracking. Analysis of diffusion and flow in two-dimensional systems. Biophys. J. 60, 910-921.

(57) Suresh, A. K., Pelletier, D. A., Wang, W., Morrell-Falvey, J. L., Gu, B., and Doktycz, M. J. (2012) Cytotoxicity induced by engineered silver nanocrystallites is dependent on surface coatings and cell types. Langmuir 28, 2727-2735.

(58) El Badawy, A. M., Silva, R. G., Morris, B., Scheckel, K. G., Suidan, M. T., and Tolaymat, T. M. (2011) Surface charge-dependent toxicity of silver nanoparticles. Environ. Sci. Technol. 45, 283-287.

(59) Nangia, S., and Sureshkumar, R. (2012) Effects of nanoparticle charge and shape anisotropy on translocation through cell membranes. Langmuir 28, 17666-17671. 